This is the final peer-reviewed accepted manuscript of:

Riccardo Accorsi, Susan Cholette, Riccardo Manzini, Alessandro Tufano, A hierarchical data architecture for sustainable food supply chain management and planning, Journal of Cleaner Production, Volume 203, 2018, Pages 1039-1054, ISSN 0959-6526

The final published version is available online at:

http://dx.doi.org/10.1016/j.jclepro.2018.08.275

Rights / License:

The terms and conditions for the reuse of this version of the manuscript are specified in the publishing policy. For all terms of use and more information see the publisher's website.

This item was downloaded from IRIS Università di Bologna (https://cris.unibo.it/)

When citing, please refer to the published version. 


\section{Accepted Manuscript}

A hierarchical data architecture for sustainable food supply chain management and planning

Riccardo Accorsi, Susan Cholette, Riccardo Manzini, Alessandro Tufano

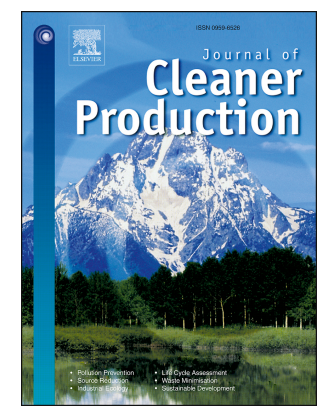

PII:

S0959-6526(18)32626-X

DOI:

10.1016/j.jclepro.2018.08.275

Reference: JCLP 14059

To appear in: Journal of Cleaner Production

Received Date: 12 February 2018

Revised Date: 13 August 2018

Accepted Date: 26 August 2018

Please cite this article as: Accorsi R, Cholette S, Manzini R, Tufano A, A hierarchical data architecture for sustainable food supply chain management and planning, Journal of Cleaner Production (2018), doi: 10.1016/j.jclepro.2018.08.275.

This is a PDF file of an unedited manuscript that has been accepted for publication. As a service to our customers we are providing this early version of the manuscript. The manuscript will undergo copyediting, typesetting, and review of the resulting proof before it is published in its final form. Please note that during the production process errors may be discovered which could affect the content, and all legal disclaimers that apply to the journal pertain. 


\title{
A hierarchical data architecture for sustainable food supply chain management and planning
}

\author{
Accorsi Riccardo ${ }^{a, *}$ \\ Cholette Susan ${ }^{\mathrm{b}}$ \\ Manzini Riccardo ${ }^{\text {a }}$ \\ Tufano Alessandro ${ }^{a}$
}

${ }^{a}$ Department of Industrial Engineering, Alma Mater Studiorum - University of Bologna, Bologna, Italy

${ }^{b}$ Decision Science, San Francisco State University, San Francisco, California, USA

* Correspondig Author:

Riccardo Accorsi, Ph.D.

riccardo.accorsi2@unibo.it

ph. +390512093415

\begin{abstract}
The agro-food industry is one of the largest parts of the European Union's economy and faces economic and environmental stresses. While food traceability systems (FTSs) inform supply chain actors of product and logistical attributes, large scale implementations are scarce and are do not support active decision making. We present a framework developed for FUTUREMED project used to perform a data-driven analysis that considers both micro and macro aspects of a food supply chain (FSC). With its comprehensive multiple-depth data architecture incorporated within a tailored decision-support platform, this framework and the resulting decision-support tool is the first to move beyond simple traceability implementation to the sustainable planning of food logistics, bridging the gap between research techniques and real-world data availability. We define KPIs that measure a subset of economic and environmental factors to quantify the impact of logistical decisions. We validate the framework with the case study of an Italian fruit trader that is considering opening a new warehouse. We conclude by suggesting that this framework be applied to more complex case studies and be enhanced through including more dimensions of sustainability.
\end{abstract}

Keywords: Food operations, Sustainable Planning, Traceability, Food Supply Chain, Data architecture, IoT

\section{Acknowledgement}

This research has been developed within the European Union and Mediterranean Project FutureMED under the grant (Grant Agreement MED/2007-2013 - FUTUREMED) of the European Regional Development Fund (ERDF). This project has been supported by the Institute of Transport and Logistics (ITL) of Emilia-Romagna, whose Alberto Preti, Stefano Dondi, and Chiara Iorfida offered valuable cooperation. The authors heartily mention Stefano Soli by Ciao S.r.l., for the data provided and the fruitful comments shared during the whole project. Other companies have been involved in the data collection and need to be thanked. Specifically, Andrea Severi and Chiara Zazzini by Naturitalia Soc. coop. Agricola, Duccio Caccioni and Gianmarco Debiase by CAAB - Centro Agro Alimentare Bologna, Sandro Stefani and Andrea Lenzi by CAMST Soc. Coop. a r.l. - La Ristorazione Italiana, Enrico Silighini and Christian Donati by Granfrutta Zani, Francesco Bassi by Agrintesa soc. coop. agricola, Massimo Savini by R.L.A. s.r.l., Stefano Soli ALEGRA Soc. coop. agr., Giordano Bianconi by N.C.V., Angelo Aulicino by Interporto Bologna, Fabrizio Bugliani and Federica Montaresi by Porto La Spezia, Claudio Torchia by Gruppo CFT, Bruno Piraccini and Gianluca Amadori by Orogel S.p.A.

This project also gains some contributions, in any way, by some Engineering students at University of Bologna that contributed at gathering and manipulating data and hence deserve mention: Giulia Olivi, Simone Sassi, Federico Losappio, Elisabetta Pazzaglia, Giulia D'Addato, Simone D'Andrea, Andrea Degiorgis, Vanessa Catalano, Irene Ciliberto, Elisa Ciaccheri, and Vittoria Calfurni. 
A hierarchical data architecture for sustainable food supply chain management and planning

\section{Abstract}

The agro-food industry is one of the largest parts of the European Union's economy and faces economic and environmental stresses. While food traceability systems (FTSs) inform supply chain actors of product and logistical attributes, large scale implementations are scarce and are do not support active decision making. We present a framework developed for FUTUREMED project used to perform a data-driven analysis that considers both micro and macro aspects of a food supply chain (FSC). With its comprehensive multiple-depth data architecture incorporated within a tailored decision-support platform, this framework and the resulting decision-support tool is the first to move beyond simple traceability implementation to the sustainable planning of food logistics, bridging the gap between research techniques and real-world data availability. We define KPIs that measure a subset of economic and environmental factors to quantify the impact of logistical decisions. We validate the framework with the case study of an Italian fruit trader that is considering opening a new warehouse. We conclude by suggesting that this framework be applied to more complex case studies and be enhanced through including more dimensions of sustainability.

Keywords: Food operations, Sustainable Planning, Traceability, Food Supply Chain, Data architecture, IoT

\section{Introduction}

The agro-food industry is a leading component of the EU economy, and governments are increasingly concerned about the long term sustainability of this sector (Notarnicola et al., 2017 $7^{\mathrm{a}, \mathrm{b}}$ ). Others have addressed stressors of food system sustainability as follows. The exploitation of natural resources like water and soil (Kummu et al., 2012), resultant GHGs emissions (Sala et al., 2017), competition for land between food and biofuels (Rathmann et al., 2010; Fisher et al., 2010 a, ; Cobuloglu and Büyüktahtakın, 2015), use of genetically modified organisms (GMOs), pesticides and chemical fertilizers (Gerdes et al., 2012; Roy et al., 2009; Boye and Arcand, 2013; McLaughlin and Kinzelback, 2015) are just few of the environmental issues affecting the food ecosystems. Food sustainability has a social as well as environmental dimension: volatile prices, bottlenecks, and food waste, losses, or contamination influence negatively impact actors and stakeholders' economic wellbeing (Garrone et al., 2014; Lebersorger and Schneider, 2014; Aiello et al., 2014). Furthermore, food insecurity contributes to social problems such as hunger and obesity (Fan and Brzeska, 2016; Thomas, 2010; Sadler et al., 2016).

Although these issues independently affect the environmental, economic, and social dimensions of sustainability, they are each exacerbated by ignoring the role operations play throughout the food supply chain (FSC) and the resultant interdependencies and externalities. Better information is crucial for improving food sector sustainability, and others (Hu et al., 2013; Etemadnia et al., 2015; Gwanpua et al., 2015) have demonstrated how increased information can drive such improvements. Higher visibility and transparency of food operations would enable precise performance assessments, resulting in more accurate planning and control of the production and distribution activities to the benefit of all stakeholders (Aiello et al., 2015; Accorsi et al., 2018). Food traceability systems (FTSs) inform consumers and other supply chain actors of product attributes, shelf-life, and logistics aspects (e.g., delivery time) (Dabbene and Gay, 2011). Although FTSs could enhance knowledge about the FSC, barriers limit their diffusion (Bosona and Gebresenbet, 2013). Implementing a traceability system is expensive 
thousands of small actors that are neither horizontally nor vertically integrated. Lastly, information uncertainty couples with a lack of standardized data acquisition and communication protocols. Thus, effective implementations of FTSs are rare.

Encouraging the diffusion of FTSs to shed light on FSC ecosystems requires specifying which information to track (Tsolakis et al., 2014). This paper illustrates such a framework for FSC data collection that builds a structured knowledge on food processes and supports integrated, data-driven planning. The underlying work is from the European Union and Mediterranean Project FUTUREMED (MED/2007-2013 - FutureMED) under the grant of the European Regional Development Fund. Their goal is mapping and optimizing agro-food supply chains for generic food products (i.e. both raw and processed) in the Mediterranean region through adopting Information and Communications Technology (ICT) in the agro-food sector, connecting supply chains actors. Note the proposed framework is intended for generic food products but remain open to others. Indeed, the characteristic that makes food supply chains distinctive and challenging even more than perishability is the extreme fragmentation of the sector, which counts hundreds of thousands of small producers who supply tens of thousands of intermediate players, who, in turn, serve thousands of selling points and hundreds of retailers, each owning a distribution chain. Furthermore, this hierarchy couples with that of decision-makers and of macro-micro issues which reflects the strategic, tactical and operational nature of supply chain planning. In such highly spread and fragmented environment, typical approaches to FTS are infeasible without first providing a comprehensive and integrated data architecture to cope with different processes to track and decision levers to handle. This assumption motivated our research.

The remainder of this paper is organized as follows. Section 2 summarizes a literature review. Section 3 defines the framework and the associated database for FSC data collection, storage, and manipulation. Section 4 illustrates how the framework enables data-driven analysis and supports decision-making for a case study. Section 5 concludes the paper by discussing the obtained results and considers potential further applications for the framework.

\section{Literature review}

Considering the impact of the food industry on the European and Mediterranean economies and the associated externalities (Kucukvar and Samadi, 2015), operations researchers have contributed in supporting decision-making on FSC management (Ahumada and Villalobos, 2009). However, these contributions separately address various decisions (i.e. of strategic, tactical and operational nature), and the need for a more unified approach is highlighted (Akkerman et al., 2010).

Indeed, classic operations research approaches inherently have limitations in solving FSC issues. The first limitation is methodological: both optimization and simulation techniques build upon a conceptual model of the system to be analyzed. Such models simplify the real system through a subset of parameters and decision variables that are exclusive and localized rather than inclusive and systemic. Secondly, the micro (localized and short term) and macro (diffused and mid-long term) perspectives of planning are rarely integrated in the same decision problem (De Keizer et al., 2017, Manzini et al., 2014 , Mason et al., 2015; Accorsi et al., 2015 and 2017 , Soto-Silva et al., 2016). Thirdly, the dearth of information availability over wider spatial scales and multiple FSC stages and stakeholders impedes properly quantitative decision-support systems. These three factors contribute to a single-minded focus on sub-optimizing parts of these complex ecosystems without considering the whole FSC (Higgings et al., 2010). 
The poor coordination among the food actors in the FSC (Handayati et al., 2015) and paucity of ICT infrastructures results in limited visibility of processes to control and prevents practical implementation of data-driven planning methodologies. Others (Aung and Chang, 2014; Ting et al., 2014) state the need for FTS frameworks for data tracking and manipulation at different stages of the FSC.

FTSs are implemented for different purposes: to comply with mandatory regulations, to attest product origin, to certify specific food properties, and to aid recall actions in case of sanitary outbreaks (Dabbene et al., 2014). Saak (2016) formulates an equilibrium model to investigate why and when a supply chain should invest in traceability systems to improve its reputation for high quality processes. Dai et al. (2015) build upon the axiomatic design method (Suh, 2001) to study the impacts of introducing a traceability system on the supply chain actors' economic interests. Aiello et al. (2015) introduce a mathematical approach to predict the profit of introducing an FTS in fruit and vegetable supply chains. Asioli et al. (2014) define a model incorporating three indices of traceability and use regression analysis to test empirically how they affect a fish processor. Dabbene and Gay (2011) formulate an optimization model to maximize FTS performance to support recalls for sanitary outbreaks. The identification of the proper trade-off between the depth and scale of a data acquisition and communication infrastructure, the associated costs, and the resulting benefits, remains challenging (Badia-Melis et al., 2015). A better understanding how a FTS can impact the performance of the FSC is necessary (Bosona and Gebresenbet, 2013).

Other researchers have worked towards the definition of frameworks that support FTSs (Regattieri et al., 2007). A search on Web of Science - Scopus from 2000-2018 for articles mentioning food supply chain and data yields 371 records, of which 56 include the term traceability and 11 also include the term framework. These 11 papers are classified in Table 1 which indicates whether the framework focuses on IT architectures, records integration from different sources or tracking sensors, is concerned on safety control or standard compliance, or supports decision making on FSC operations and layers.

Some of these deserve mention. Bechini et al. (2008) introduce a data model for traceability that describes FCS assets and explore the behavioral patterns of a food lot throughout the distribution pipeline. Li et al. (2010) illustrate a framework for early warning systems using data mining methods applied to a case study of a chicken slaughterhouse. Storøy et al. (2013) present the TraceFood framework to facilitate data interchange through a non-proprietary standard and outline best practices for the implementation of a FTS. Manzini and Accorsi (2013) propose a framework to ensure the quality of products throughout logistical operations. Ting et al. (2014) incorporate data-mining approaches into a decision-support tool to address safety issues. Pizzuti et al. (2014) illustrate a food Track \& Trace (T\&T) ontology to aid the conceptualization, design, and implementation of FTSs, while Pizzuti and Mirabelli (2015) present a T\&T system based on a comprehensive food value chain model.

Although FTSs track lot-specific details throughout the FSC, few tools are able to infer product flows along complex, large scale supply chain networks. Within the FRISBEE European project Gogou et al. (2015) and Gwanpua et al. (2015) both illustrate a web-based platform to collect and analyze the temperature conditions throughout chilled and frozen FSCs. Verdouw et al. (2016) demonstrate virtualizing a FSC according to the Internet-of-things paradigm and provide an enabling information system architecture. The main contribution documented is a national database 
FSCs. Compared to LeBlanc et al.'s work, this paper proposes a database and an integrated decision-support tool for the mapping, planning, and optimization of food production and distribution operations in the Mediterranean region. Given the properties of a traceability system defined by McEntire et al. (2010), the framework proposed in this paper is a flexible, large breadth, geo-referenced, and multiple-depth data architecture to enable data-driven analysis and planning of food logistics operations. It is flexible because it is not just a data repository for FTSs, but it also behaves as dataset to design, analyze, and optimize operations. It has large breadth since it refers to physical flows of products from farm-to-table and includes characteristics of entities involved at each stage including geo-referencing. Lastly, a multiple-depth approach integrates data layers (i.e., micro and macro) and granularities, and enables the linkage of different abstraction levels and records throughout the FSC system.

To our knowledge, this paper covers a gap in the literature that lacks of holistic decision-support systems addressing simultaneously to macro and micro issues on food supply chain planning and management. We propose an original ontological framework and a support-decision platform aimed beyond just traceability implementation to the datadriven planning of food production and distribution operations over a large scale environment.

\section{Methodology and framework design}

We build a framework in six steps:

1. Step 1: Modelling the FSC boundaries and the decision-makers;

2. Step 2: Analyzing the FSC's entities;

3. Step 3: Designing the data architecture and its database;

4. Step 4: Collecting and aligning data;

5. Step 5: Developing a decision-support tool and interfaces;

6. Step 6: Validating and applying the framework.

The first step conceptualizes the FSC operations by depicting the activities and associated actors necessary to process and distribute food from growers/farmers to the consumers. We identify the nodes/stages where tasks occur, the decision-makers involved, along with the information abstraction layers and system boundaries required for holistic planning. An information abstraction level represents a layer of analysis, while boundaries highlight which food processes or physical and information flows are mapped.

The second step defines the FSC's entities, identifying a panel of properties and features for each that characterize its role in the FSC's operations. This step assesses the relevance of each entity in accordance with its data abstraction level and determines the relational linkages. In our framework, such entities are the information holders or the physical objects to which data records are referred.

The third step designs a flexible database for the analysis of FSC operations. It allows for collection of localized micro FTS records at a low level of abstraction, and organization of information belonging to different abstraction levels through a multiple-depth integration. Integration between information layers is obtained through a hierarchical data architecture which combine the stages of the FSC and the related entities with the data tracked to support decisionmaking as illustrated in Fig. 1. Data integration follows two approaches: the top-down inference uses data available at an higher level (e.g. seasonal food consumption per province) to infer records at lower level (e.g. physical food flows handled by the nodes of different distribution channels within a province); the bottom-up inference uses records tracked 
at lower lever (e.g. the environmental stresses measured along a shipment) to deduce the behavior of a process or an entity at higher levels (e.g. stresses experienced by food on a route and given season). The proposed data architecture results into a comprehensive database which links the stages of a generic FSC and collects records over European scale. The fourth step finalizes an extensive data collection from different and multiple-depth sources, including national and European statistics databases, existing Geographic Information Systems (GISs), Information Systems of the involved companies, handbooks, and websites. This step also aligns the space and time granularity of the available information. The resulting relational database is filled with primary and secondary data. The fifth step develops a decision-support platform and user interfaces for data-driven planning and analysis of FSC operations. The developed tool is intended to addressed to different planning targets in agreement with the purposes of different decision-makers as exemplified in Fig. 1. The tool virtualizes the physical flows of food throughout existing or new supply chains, then quantifies and assesses a panel of economic, environmental, and logistics KPIs, compares alternative network scenarios through what-if multi-scenario analysis, and runs optimization models. The final step validates the framework with a proof of concept used to address to a company's issue.

The resulting database and tool support the collection and organization of food related data at multiple abstraction levels, the mapping of the flows for a generic FSC over a large scale, the analysis of food operations and performances of involved nodes and actors, and the optimization of punctual processes rather than the overall pipeline from farm-totable. The most important activities for each step are described in the following sections, while the conceptual framework with the data abstraction levels, entities, decision-makers, planning targets and boundaries is illustrated in Fig. 1.

Fig. 1. Framework targets, boundaries and decision-flows.

\subsection{Modelling the FSC boundaries and decision-makers}

A FSC involves all the stages from farmers to consumers. In order to understand the information to track and collect at each stage we adopted an approach known as Material Flow Analysis (MFA), aimed at drawing the physical food flows throughout the supply chain. The resulting key information to collect is the handled goods at each stage, the actors and decision-makers involved, the processes carried out, and the node where the process is performed. The goods flow is characterized by a food product and handled volume. The type of product ranges from raw food (e.g. fruits and vegetables) and agro-food commodities, semi-processed foods, bulk or packaged finish foods, and the proper unit of measure for the volume varies accordingly.

As illustrated in Fig. 1., the framework couples three different hierarchies. The first deals with the decision-makers and stakeholders involved in FSC planning which range from growers, distributors and retailer, to regulatory agencies and bureaus of agriculture, infrastructures and transportation. The second refers to the records and sets of data required by decision-makers to handle and take data-driven operational, tactical or strategic decisions. The classification that follows cross-references the first two hierarchies taking inspiration from others' taxonomies (Tsolakis et al., 2014; Pizzuti and Mirabelli, 2015; Accorsi et al., 2017 ): cultivating and farming. To plan seeding, cropping, harvesting or farming operations the agro-climate profiles 
(i.e. soil and weather features) of crop/farm nodes, the cost of fertilizers and nutrients, and the suppliers portfolio and demand need to be collected.

- Suppliers/Producers/Packagers collect and consolidate raw products from the growers behaving as

The places where the aforementioned decision-makers/actors operate represent logistics nodes or stages of the FSC network, and some of these identify physical points for tracking and allocating flows. The combination of the first two hierarchies of Fig. 1 is justified by the nature of planning: at bottom levels many operational decisions can be taken each fueled by wide sets of parameters and information; at top level, given the complexity and impact of decisionmaking, this should be supported by synthetic but comprehensive KPIs.

The vast number of actors involved in transnational geographic distribution typically discourages the implementation of T\&T protocols and technologies. Thus, information on food flows is limited for some stages, actors, or nodes. Nevertheless, the planning of sustainable food operations requires integrated data-driven analysis fueled by a comprehensive dataset. The proposed framework identifies, in the third hierarchy, three abstraction levels for data collection and shows the links and interdependencies between information layers, allowing for inferring missing records though an integrated top-down and bottom-up approach for the analysis of the food operations over a large scale geography. These are represented in Fig. 1 and described as follows:

- Geographical level refers to the panel of high-level/strategic parameters describing the role of a geographical area, to assess its potential for the development of agro-food supply chains, and to compare this potential with those of other areas. This panel includes quantitative metrics that describe the economics, logistical 
performances, level of maturity of the agricultural sector, available transport infrastructure, and environmental profile. The import-export balance, production volume, and flows of agro-food products distributed between

The identification of these abstraction levels, which borrow the levels of planning i.e. strategic, tactical and operational, shows how traceability systems alone are not sufficient to support the systemic planning of FSC operations as they focus on a single process and do not integrate systemic information.

\subsection{The FSC entities}

The entities defined at Step 2 represents physical objects, processes, and nodes. Each entity belongs to an abstraction level characterized by the set of parameters and data collected at that level. Fig. 2 illustrates the role of the main entities, their connections, and the knowledge hierarchy through the three abstraction levels via Object-Relational Mapping (ORM) notation,

The entity Geoarea represents a territory where parameters describing the climatic, socio-economic, infrastructural, agricultural, or logistical profiles are known. These quantitative properties couple with the flows between two Geoareas: demands, supply capacities, and the import-export balance for each food product. The granularity of Geoareas is not predetermined, ranging from a city to a country depending on information availability. The entity Node represents a FSC node and is characterized by parameters describing its operational role. The connection between a couple of Nodes is given at the Logistics level via the Shipment entity and is typically characterized by origin and destination nodes, a departure date, a load to be delivered, and Transport vehicle. A Shipment is organized in response to an Order by a Customer.

The properties of these entities can be further explored On-field through use of sensors able to collect soil/climate features of a crop (i.e. soil and micro-climate profiles in Fig. 2), timely information on a single product or unit load (i.e., transportation profile in Fig. 2), or track primary production, processing activities, and distribution processes.

Fig. 2. Framework entities

\subsection{Data architecture}

The hierarchy of relevant entities and associated data and parameters identified in the framework defines the relational database tables for collecting, storing, and manipulating the records describing the characteristics and role of each entity 
coordinates, the address, and binary parameters tracking the node's access to a specific transport mode. The last parameters indicate the node's potential for exploiting transshipments. The classification given in Section 3.1 results in defining different tables for separate roles, avoiding field redundancies and sustaining table normalization. The tables Crop, Processing Plant, Storage Facility, Port, Multimodal hub, and Market further define each node. For example, a Crop table record reports the number of hectares, the variety cultivated, the set of irrigation and harvesting systems applied, and at the On-field level the soil and the micro-climate profiles (Accorsi et al., 2016). A record from the table Storage Facility includes typical warehouse design parameters, storage equipment, the number of aisles, bays and storage level, and temperature set-points of cold rooms.

According to the hierarchy of Fig. 2, each node belongs to a GeoArea, which behaves as a collector of nodes. The framework organizes the set of import and export rules and certifications required to distribute different food categories among GeoAreas of different countries. The table Flow tracks physical flows of food distributed from a GeoArea to another over time. At a lower level, the table Shipment tracks physical deliveries among nodes by recording the origin and destination, departure date, shipped products, and transport mode. The Product table records properties of products: product category, variety, nutritional values, and links to shelf life profiles and conservation requirements. These tables permit tracking the environmental profile of a shipment in term of monitored temperature and humidity records, and the combination of package and product that have that profile at the On-field level. These records can be used for laboratory simulations testing the responsiveness of both product and package to transport conditions and measuring the impact of logistics processes on the product's quality and safety (Manzini et al., 2014 ${ }^{\mathrm{b}}$; La Scalia et al., 2016; Ayyad et al., 2017; Valli et al., 2013). Table 2 shortlists the tables and their main fields and quantifies the number of records collected per table.

Fig. 3. Database E-R diagram.

While the framework is generalizable to all FSCs, we focus on fruit and vegetable supply chains. The data sources used to populate the tables of the geographical level include annual authorities' reports, whitepapers, websites, and a thesaurus of databases. The gathering of lower level records has been supported by a group of enterprises involved in the FutureMed project that provided primary data from ERP systems, questionnaires to managers at various nodes, and information from websites. The sources for On-field information are mainly storage and distribution processes of specific food companies that have been monitored via environmental sensors.

Table 2. Database fields, abstraction levels (AL), and records number.

\subsection{Decision-support tool and interfaces}

We developed a decision-support platform (DST) to address the following research goals (RGs) and planning targets. The DST works via desktop software developed in C\# .NET and interfaces with a MS SQL Server database while reading other data sources such as MS Access databases or Excel files, and it uses MS Mappoint and OpenStreetMap to provide maps and retrieve point-to-point routing information.

- $\quad R G$ 1: Overviewing the information at different abstraction level with a GIS support, and performing queries to the database;

- $\quad R G$ 2: Inferring the missing information at lower (higher) levels through a top-down (bottom-up) approach; 
- $\quad R G$ 3: Quantifying a panel of economic, logistics and environmental KPIs resulting by the food production and distribution scenario (as-is) throughout the FSC;

- $\quad R G$ 4: Setting different parameter-driven scenario (to-be) of a FSC and comparing the associated performances in what-if simulation analysis;

- $\quad R G 5$ : Creating and applying models to optimize production-distribution scenarios (optimized to-be) from a holistic perspective.

The list of RGs couples with some FSC planning targets as shown in Fig. 1. Some of the tools' Graphic User Interfaces (GUIs) follow. $R G 1$ is met by the GUI that summarizes records collected at Geographical and Logistics levels by mapping FSC nodes and uses colors and size to compare and quantify the desired metrics, as shown in Fig. 4. By achieving $R G$ 1, the decision-makers at different levels of the hierarchy can identify business opportunities (e.g. uncovered markets, production areas close to logistics infrastructures) or assess the utilization of existing logistics infrastructures (e.g. storage facilities, ports).

Fig. 4. Tool GUIs: Overviewing the high-level FSC records with a GIS.

A twofold approach is implemented for $R G 2$. Information at the higher abstraction level infers the shared behavior of the nodes that belong to a GeoArea via a top-down approach. For example, the physical flow of apples distributed from one region to another are tracked in the table Flow letting us assume that the storage facilities and ports located within those areas are responsible for such flows. Thus, this flow can be split among these nodes, and distribution records at logistics level can be estimated and appended to the Shipment table. The DST integrates records at all three levels to determine food flows throughout the FSC. The annual flow of a product among GeoAreas can be allocated to growers, processing facilities, ports, storage facilities, and market nodes of those areas. The more collected nodes, the more reliable flow allocations will be. The user can estimate a product's seasonal demand given a region's market nodes as illustrated in Fig. 5. Should Shipment profiles be available, the DST would use this information to infer the environmental conditions experienced during the distribution of food from one GeoArea to another. Thus, the DST

By achieving $R G 2$, the decision-maker deepen its knowledge of the FSC operations and also understand where gaps in data collection exist and which processes are hence out-of-monitoring.

RGs 3 and 4 are met though the GUIs illustrated in Fig. 6, which allow for: (1) assessing physical food flows between the nodes as recorded in the table Shipment; (2) analyzing an as-is distribution scenario in term of quantitative economic, logistics, and environmental metrics; (3) generating a set of to-be distribution scenarios for what-if comparisons; and (4) deciding location-allocations across the FSC by virtualizing parameter-driven to-be scenarios. A panel of KPIs compare distribution scenarios throughout the FSC. Fig. 6 shows the map of the food shipments through a selected network, the environmental GHGs associated with the trucks, and the frequency analysis of the distance travelled per shipment. Furthermore, the GUI uses colors to compare two distribution scenarios, orange for as-is and blue for to-be, plotting shipments in term of travelled distance and utilization. RGs 3 and 4 address to several planning targets as transport cost reduction, to the assessment of environmental externalities of food operations, and to the redesign of the network infrastructure. 
Lastly, $R G 5$ aims at designing optimized to-be FSC scenarios through strategic, tactical and operational optimization models. For sake of brevity, the formulation of such models, as well as their application with the data architecture of the FUTUREMED project, is devoted to other and future papers.

Fig. 6. Tool GUIs: Distribution scenarios and KPIs assessment.

\section{Proof of concept}

The decision-support platform has been tested with industrial case studies, each addressing to different $R G s$ and planning targets, some of which will be presented in future papers. One such case study concerns the distribution scenario of an Italian fruit trader that is assessed and improved by addressing to the RGs 1, 3 and 4. This Italian SME operating in the food import/export across the Mediterranean purchases fruit from consolidators and processors and then arranges distribution activities to customers. As the company is committed to daily negotiations of purchasing and selling prices, picking and delivery tours, and filing import-export documents, the proposed DST aids by providing information such as transport costs between nodes, country-specific rules, area food demand (See Fig. 8), and an overview of the opportunities for improving logistics costs. Indeed, we adopted the proposed framework and DST to virtualize the existing (as-is) distribution scenario and try the impact of a new storage facility located in Vignola (MO). "Is that location convenient?", and "which are the economic and environmental returns from the new facility?" are two questions addressed by our framework. Fig. 7 summarizes the main company's network features and characteristics and shows the nodes of the observed FSC on the map.

Fig. 7. Company's network features and map.

The DST has been used to support the company's decision to establish a storage facility as intermediate stage between the suppliers and the customers. The new facility would consolidate shipments, leading to better food conservation and decreasing food miles. The tool creates both $a s$-is and to-be distribution scenarios, comparing them via a panel of economic, logistics and environmental metrics. Two destination markets, Germany and Unit Arab Emirates, are also considered, and new supply chains connecting these nodes with the existing suppliers' network have been assessed with the tool.

Fig. 8. Customer demand for oranges, peaches and apples in German markets.

The Flow records at the geographical level estimate the demand per product and city for both customers' markets (see Fig. 8). Data provided by the company virtualizes the network, calculates the routes, quantifies the seasonal volume of fruit retrieved by processors and delivered to customers, and then estimates the needed storage capacity of the new warehouse at the logistics level. The fruit volume varies by season but is equal in both scenarios that differ only by whether a new storage facility exists. A list of shipments over an annual horizon and a panel of KPIs are generated for each distribution scenario.

Fig. 9. Tool GUIs: Company testbed 
The as-is scenario uses two storage facilities in Albenga and Verona to consolidate the shipments, while the to-be scenario includes a new warehouse located in Modena surroundings (i.e., Vignola as in Fig. 9) suggested by the company's managers. The distribution scenarios follow two stages: first from the producer to the storage facility and then to the customer. Starting from the annual orders profiles, the routes, from the suppliers to the warehouse and then to the consumers, have been virtualized by the DST and distances calculated to minimize travelling for each shipment. The new storage facility aims at consolidating the shipments and at reducing the travelling distance from suppliers to consumers' nodes.

Table 3. What-if analysis with the Company testbed.

Table 3 quantifies a panel of some economic, logistics and environmental KPIs (i.e. addressing to $R G 3$ ) and summarizes the results from the what-if comparison analysis between the as-is and to-be scenarios. The new warehouse in the to-be scenario would reduce the overall total travelled distance for food distribution, which consequently decreases both transport costs and resulting environmental externalities. The shipments in the to-be scenario are lower because the warehouse enhances the consolidation of demand orders. Dealing with logistics, the DST quantifies the economic saving per year between as-is and to-be scenario in almost $45 \mathrm{k} €$ as calculated from the average transport cost reduction multiplied the number of shipments. This benefit provided valuable information for deciding whether to invest in the new storage facility and how long payback would take, while the other changes in the environmental metrics quantifies the GHG emissions savings that would be possible (i.e., $8 \%$ reduction), which may be useful for capand-trade and emission mitigating systems as EU ETS or white certificates (Convery, 2009; Sorrell et al., 2009).

\section{Discussion and future developments}

Now that the framework and database have been developed and validated through a case study, we consider potential applications and avenues for future development. The most strategic level would entail the planning and optimization of the European and Mediterranean food production and distribution systems while considering long-term sustainability. One use-case could be assessing the environmental externalities from the distribution flows of food across the countries. Another analysis might entail investigating ports (Bartholdi et al., 2016), warehouses, transshipment hubs and other logistics infrastructures with respect to current utilization and the opportunities for improvement. Further down the hierarchy of decision levels the database and DSTs would be useful in identifying distribution bottlenecks and suggesting the most feasible and sustainable food routes given both economic and environmental aspects. Another use would be identifying new market opportunities given existing logistics connections, product shelf lives, availability of refrigerated infrastructure (Gallo et al., 2017), and distances between suppliers and consumers, based on aggregate purchasing power and market demand. At the most operational level, these tools could work within the Internet-ofThings paradigm as detailed by Accorsi et al., $\left(2017^{\mathrm{b}}\right.$ ) and yet exemplified by others (Yan et al., 2016; Zhang et al., 2017; Li et al., 2017), allowing for adjustments at the farm, processing and distribution levels, given based on a variety of micro data such rainfall patterns and vendor costs.

Thus, we anticipate using this framework to illustrate other more complex case studies such as the re-design of a retailer supply chain for fruit and vegetable products, the assessment of ready-to-eat vegetables' deliveries to tourism areas, and the optimization of shipping routes in the Mediterranean basin, according to the food seasonality and shelf-life. All of these studies would simultaneous provide a more a detailed description of each DST and the related functionalities available in the tool set. 
While many applications are possible with the current functionality of the database and supporting DST, we also consider future improvements. Table 3 addresses key logistical, economic, and environmental metrics, specifically greenhouse gas emissions. Of course, food system sustainability encompasses more that this subset of drivers. Extensions to the framework and supporting database should include other environmental factors such as land use issues, as is done in Accorsi et al. (2016), and social factors: for example, such extensions could support community planners' efforts to ensure citizens' access to quality food.

A future extension would entail using these results to educate supply chain actors and even ultimately consumers of the externalities associated with hidden operations from farm-to-table, thereby eliciting more sustainable choices (Cholette et al., 2013). For instance informed consumers' aversion to palm oil due to environmental externalities (The Guardian, 2015; Huffington, 2017), has resulted in Italian retailers stocking fewer such products.

\section{Conclusion}

This paper illustrates a framework for the design of a database aiding the assessment, planning and design of food production and distribution operations over a large scale area and strategic perspective. To the authors' knowledge, this framework and the resulting decision-support platform is the first to move beyond simple traceability implementation to the sustainable planning of food logistics operations over a large-scale environment that bridges the gap between research techniques and real-world data availability.

This database and the associated DSTs support researchers and practitioners alike in collecting and organizing the information necessary for a quantitative analysis and assessment. For example, in the aforementioned case study for the Italian SME importer/exporter, the economic savings associated with the logistics operations provided valuable information for deciding whether to invest in improving the logistic network and quantifying the resultant environmental benefits. The database and DSTs could be used as-is to inform decisions made at all levels in the FSC or could be further extended to consider additional dimensions of sustainability.

\section{Acknowledgement}

\section{References}

Accorsi, R., Cholette, S., Manzini, R., Pini, C., Penazzi, S., 2016. The Land-Network problem: Ecosystem carbon balance in planning sustainable agro-food supply chains. Journal of Cleaner Production 112(1), 158-171.

Accorsi, R., Gallo, A., Manzini, R., 2017a. A climate-driven decision-support model for the distribution of perishable products. Journal of Cleaner Production 165, 917-929.

Accorsi, R., Baruffaldi, G., Manzini, R., Tufano, A., 2018. On the design of cooperative vendors' networks in retail food supply chains: A logisticsdriven approach. International Journal of Logistics Research and Applications, 21(1), 35-52.

Accorsi, R., Bortolini, M., Baruffaldi, G., Pilati, F., Ferrari, E., 2017 ${ }^{\mathrm{b}}$. Internet-of-things paradigm in food supply chains control and management. Procedia Manufacturing 11, 889-895.

Accorsi, R., Manzini, R., Pini, C., Penazzi, S., 2015. On the design of closed-loop networks for product life cycle management: Economic, environmental and geography considerations. Journal of Transport Geography 48, 121-134.

Ayyad, Z., Valli, E., Bendini, A., Accorsi, R., Manzini, R., Bortolini, M., Gamberi, M., Gallina Toschi, T., 2017. Simulating international shipments of vegetable oils: Focus on quality changes. Italian Journal of Food Science 29, 38-49.

Ahumada, O., Villalobos, J.R., 2009. Application of planning models in the agro-food supply chain: A review. European Journal of Operational Research 196(1), 1-20. 
Ahumada, O., Villalobos, J.R., 2011. Operational model for planning the harvest and distribution of perishable agricultural products. International Journal of Production Economics 133(2), 677-687.

Aiello, G., Enea, M., Muriana, C., 2014. Economic benefits from food recovery at the retail stage: An application to Italian food chains. Waste Management 34(7), 1306-1316.

Aiello, G., Enea, M., Muriana, C., 2015. The expected value of the traceability information. European Journal of Operational Research 244, $176-186$.

Asioli, D., Boecker, A., Canavari, M., 2014. On the linkages between traceability levels and expected and actual traceability costs and benefits in the Italian fishery supply chain. Food Control 46, 10-17.

Akkerman, R., Farahani, P., Grunow, M., 2010. Quality, safety and sustainability in food distribution: a review of quantitative operations management approaches and challenges. OR Spectrum 32, 863-904.

Aung, M.M., Chang, Y.S., 2014. Traceability in a food supply chain: Safety and quality perspectives. Food Control 39, $172-184$.

Badia-Melis, R., Mishra, P., Ruiz-Garcia, L., 2015. Food traceability: New trends and recent advances. A review. Food Control 57, $393-401$.

Bartholdi, J.J. Jarumaneeroj, P., Ramudhin, A., 2016. A new connectivity index for container ports. Maritime Economics 18, $231-249$.

Bechini, A., Cimino, M.C.G.A., Marcelloni, F., Tomasi, A., 2008. Patterns and technologies for enabling food supply chain traceability throughout collaborative e-business. Information and Software Technology 50, 342-359.

Boye, J.I., Arcand, Y., 2013. Current trends in green technologies in food production and processing. Food Engineering Reviews 5(1), 1-17.

Bosona, T., Gebresenbet, G., 2013. Food traceability as an integral part of logistics management in food and agricultural supply chain. Food Control $33,32-48$.

Cholette, S., Ungson, G.R., Özlük, Ö., Özşen, L., 2013. Exploring purchasing preferences: Local and ecologically labelled foods. Journal of Consumer Marketing, 30(7), 563-572.

Cobuloglu, H.I., Büyüktahtakın, I.E., 2015. Food vs. biofuel: An optimization approach to the spatio-temporal analysis of land-use competition and environmental impacts. Applied Energy 140, 418-434.

Convery, F.J., 2009. Origins and development of the EU ETS. Environmental and Resource Economics, 43(3), 391-412.

Dabbene, F., Gay, P., 2011. Food traceability systems: Performance evaluation and optimization. Computers and Electronics in Agriculture 75, 139146.

Dabbene, F., Gay, P., Tortia, C., 2014. Traceability issues in food supply chain management: A review. Biosystems Engineering $120,65-80$.

Dai, H., Ge, L., Zhou, W., 2015. A design method for supply chain traceability systems with aligned interests. International Journal of Production Economics 170, 14-24.

De Keizer, M., Akkerman, R., Grunow, M., Bloemhof, J.M., Haijema, R., van der Vorst, J.G.A.J., 2017. Logistics network design for perishable products with heterogeneous quality decay. European Journal of Operational Research 262, 535-549.

Etemadnia, H., Goetz, S., Canning, P., Tavallali, M.S., 2015. Optimal wholesale facilities location within the fruit and vegetables supply chain with bimodal transportation options: An LP-MIP heuristic approach. European Journal of Operational Research 244, 648-661.

Fan, S., Brzeska, J., 2016. Sustainable food security and nutrition: Demystifying conventional beliefs. Global Food Security 11, 11-16.Fischer, G., Prieler, S., van Velthuizen, H., Lensink, S.M., Londo, M., de Witc, M., 2010. Biofuel production potentials in Europe: Sustainable use of cultivated land and pastures. Part I: Land productivity potentials. Biomass and Bioenergy 34, 159-172.

Farahani, P., Grunow, M., Akkerman, R., 2013. Design and operations planning of municipal foodservice systems. International Journal of Production Economics 144, 383-396.

Fischer, G., Prieler, S., van Velthuizen, H., Berndes, G., Faaij, A., Londo, M., de Witc, M., 2010. Biofuel production potentials in Europe: Sustainable use of cultivated land and pastures, Part II: Land use scenarios. Biomass and Bioenergy 34, 173-187.

Gallo, A., Accorsi, R., Baruffaldi, G., Manzini, R., 2017. Designing sustainable cold chains for long-range food distribution: Energy-effective corridors on the Silk Road belt. Sustainability, A Journal 2017, 9(11), 2044; doi:10.3390/su9112044.

Gerdes, L., Busch, U., Pecoraro, S., 2012. GMOfinder - A GMO screening database. Food Analytical Methods 5, $1368-1376$.

Gwanpua, S.G., Verboven, P., Leducq, D., Brown, T., Verlinden, B.E., Bekele, E., Aregawi, W., Evans, J., Foster, A., Duret, S., Hoang, H.M., van der Sluis, S., Wissink, E., Hendriksen, L.J.A.M., Taoukis, P., Gogou, E., Stahl, V., El Jabri, M., Le Page, J.F., Claussen, I., Indergård, E., Nicolai, B.M., Alvarez, G., Geeraerd, A.H., 2015. The FRISBEE tool, a software for optimising the trade-off between food quality, energy use, and global warming impact of cold chains. Journal of Food Engineering 148, 1-12.

Handayati, Y., Simatupang, T.M., Perdana, T., 2015. Agri-food supply chain coordination: the state-of-the-art and recent developments. Logistics Research 8(5), 1-15.

Higgins, A.J., Miller, C.J., Archer, A.A., Ton, T., Fletcher, C.S., McAllister, R.R.J., 2010. Challenges of operations research practice in agricultural value chains. Journal of the Operational Research Society 61(6), 964-973.

Hu, J., Zhang, X., Moga, L.M., Neculita, M., 2013. Modeling and implementation of the vegetable supply chain traceability system. Food Control 30, $341-353$. 
Huffington Post, 2017. Palm oil products are being pulled from some stores in Italy after a report linking it to cancer. Available online at: https://www.huffingtonpost.com/entry/italy-nutella-cancer_us_58792c3de4b0e58057feac94

Kucukvar, M., Samadi, H., 2015. Linking national food production to global supply chain impacts for the energy-climate challenge: the cases of the EU-27 and Turkey. Journal of Cleaner Production 108, 395-408.

Kummu, M., de Moel, H., Porkka, M., Siebert, S., Varis, O., Ward, P.J., 2012. Lost food, wasted resources: Global food supply chain losses and their impacts on freshwater, cropland, and fertilizer use. Science of the Total Environment 438, 477-489.

La Scalia, G., Aiello, G., Miceli, A., Nasca, A., Alfonzo, A., Settani, L., 2016. Effect of vibration on the quality of strawberry fruits caused by simulated transport. Journal of Food Process Engineering 39, 140-156.

LeBlanc, D.I., Villeneuve, S., Hashemi Beni, L., Otten, S., Fazil, A., McKellar, R., Delaquis, P., 2015. A national produce supply chain database for food safety risk analysis. Journal of Food Engineering 147, 24-38.

Li, Y., Kramer, M.R., Beulens, A.J.M., van der Vorst, J.G.A., 2010. A framework for early warning and proactive control systems in food supply chain networks. Computers in Industry 61, 852-862.

Li, Z., Liu, G., Liu, L., Lai, X., Xu, G., 2017. IoT-based tracking and tracing platform for prepackaged food supply chain. Industrial Management \& Data Systems 117(9), 1906-1916.

Manzini, R., Accorsi, R., 2013. The new conceptual framework for food supply chain assessment. Journal of Food Engineering 115(2), 251-263.

Manzini, R., Accorsi, R., Bortolini, M., 2014 ${ }^{\text {a }}$ Operational planning models for distribution networks. International Journal of Production Research 52(1), 89-116.

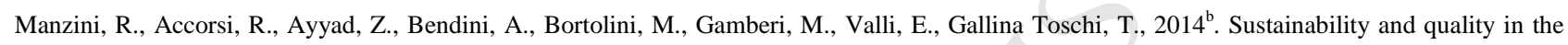
food supply chain. A case study of shipment of edible oils. British Food Journal 116, 2069-2090.

Mason, N., Flores, H., Villalobos, J.R., Ahumada, O., 2015. Planning the planting, harvest, and distribution of fresh horticultural products. International Series in Operations Research and Management Science 224, 19-54.

McLaughlin, D., Kinzelback, W., 2015. Food security and sustainable resource management. Water Resources Research 51, 4966-4985.

Notarnicola, B., Sala, S., Assumpció, A., McLaren, S.J., Saouter, E., Sonesson, U., 2017ª The role of life cycle assessment in supporting sustainable agri-food systems: A review of the challenges. Journal of Cleaner Production 140 (2), 399-409.

Notarnicola, B., Tassielli, G., Renzulli, P.A., Castellani, V., Sala, S., 2017 . Environmental impacts of food consumption in Europe. Journal of Cleaner Production 140 (2), 753-765.

Penazzi, S., Accorsi, R., Ferrari, E., Manzini, R., Dunstall, S., 2017. Design and control of food job-shop processing systems: A simulation analysis in the catering industry. International Journal of Logistics Management 28(3), 782-797.

Pizzuti, T., Mirabelli, G., Sanz-Bobi, M.A., Gomez-Gonzalez, F., 2014. Food Track \& Trace ontology for helping food traceability control. Journal of Food Engineering 120, 17-30.

Pizzuti, T., Mirabelli, G., 2015. The Global Track\&Trace System for food: General framework and functioning principles. Journal of Food Engineering 159, 16-35.

Rathmann, R., Szklo, A., Schaeffer, R., 2010. Land use competition for production of food and liquid biofuels: An analysis of the arguments in the current debate. Renewable Energy 35, 14-22.

Regattieri, A., Gamberi, M., Manzini, R., 2007. Traceability of food products: general framework and experimental evidence. Journal of Food Engineering 81, 347-356.

Roy, P., Nei, D., Orikasa, T., Xu, Q., Okadome, H., Nakamura, N., Shiina, T., 2009. A review of life cycle assessment (LCA) on some food products. Journal of Food Engineering 90, 1-10.

Saak, A.E., 2016. Traceability and reputation in supply chain. International Journal of Production Economics 177, $149-162$.

Sadler, R.C., Gilliland, J.A., Arku, G., 2016. Theoretical issues in the 'food desert' debate and ways forward. GeoJournal 81 (3), $443-455$.

Sala, S., Assumpció, A., McLaren, S.J., Notarnicola, B., Saouter, E., Sonesson, U., 2017. In quest of reducing the environmental impacts of food production and consumption. Journal of Cleaner Production 140 (2), 387-398.

Sorrell, S., Harrison, D., Radov D., Klevnas, P., Foss, A., 2009. White certificate schemes: Economic analysis and interactions with the EU ETS. Energy Policy, 37(1), 29-42.

Soto-Silva, W.E., Nadal-Roig, E., González-Araya, M.C., Pla-Aragones, L.M., 2016. Operational research models applied to the fresh fruit supply chain. European Journal of Operational Research 251, 345-355.

Storoy, J., Thakur, M., Olsen, P., 2013. The TraceFood framework - Principles and guidelines for implementing traceability in food value chains. Journal of Food Engineering 115, 41-48.

Suh, N.P., 2001. Axiomatic design: Advances and application. Oxford University Press, New York.

The Guardian, 2015. The Italians fighting against an "invasion" of palm oil. Available online at: https://www.theguardian.com/sustainablebusiness/2015/dec/09. 
Thomas, B.J., 2010. Food deserts and the sociology of space: Distance to food retailers and food insecurity in an Urban American neighborhood. World Academy of Science, Engineering and Technology 43, 19-28.

Ting, S.L., Tse, Y.K., Ho, G.T.S., Chung, S.H., Pang, G., 2014. Mining logistics data to assure the quality in a sustainable food supply chain: A case in the red wine industry. International Journal of Production Economics 152, 200-209.

Tsolakis, N.K., Keramydas, C.A., Toka, A.K., Aidonis, D.A., Iakovou, E.T., 2014. Agrifood supply chain management: A comprehensive hierarchical decision-making framework and a critical taxonomy. Biosystems Engineering 120, 47-64.

Valli, E., Manzini, R., Accorsi, R., Bortolini, M., Gamberi, M., Bendini, A., Lercker, G., Gallina Toschi, T., 2013. Quality at destination: Simulating shipment of three bottled edible oils from Italy to Taiwan. Rivista Italiana delle Sostanze Grasse 90, $163-169$.

Yan, B., Yan, C., Ke, C., Tan, X., 2016. Information sharing in supply chain of agricultural products based on the Internet of Things. Industrial Management \& Data Systems 116(7), 1397-1416.

Zhang, Y, Zhao, L., Qian, C., 2017. Modeling of an IoT-enabled supply chain for perishable food with two-echelon supply hubs. Industrial Management \& Data Systems 117(9), 1890-1905. 


\section{ACCEPTED MANUSCRIPT}

\begin{tabular}{|c|c|c|c|c|c|c|c|}
\hline Reference & $\begin{array}{c}\text { IT } \\
\text { Architecture }\end{array}$ & $\begin{array}{c}\text { Data } \\
\text { Integration \& } \\
\text { Alignment }\end{array}$ & $\begin{array}{l}\text { Regulation \& } \\
\text { standard } \\
\text { compliance }\end{array}$ & $\begin{array}{c}\text { Tracking } \\
\text { Technologies }\end{array}$ & $\begin{array}{l}\text { Food } \\
\text { Safety }\end{array}$ & $\begin{array}{l}\text { Supply Chain } \\
\text { Design \& } \\
\text { Management }\end{array}$ & $\begin{array}{c}\text { Support to } \\
\text { decision- } \\
\text { making }\end{array}$ \\
\hline Bechini et al., 2005 & $\bullet$ & $\bullet$ & ० & ○ & 0 & O & o \\
\hline Shanahan et al., 2009 & ० & ○ & - & - & ० & ० & o \\
\hline Lenton, 2010 & ० & ○ & - & & $\bullet$ & ० & o \\
\hline Costa et al., 2013 & - & ० & ० & - & ० & ० & ० \\
\hline Storøy et al, 2013 & - & $\bullet$ & ० & ○ & O & ○ & o \\
\hline Zhang et al., 2014 & ○ & ० & - & ○ & $\bullet$ & ○ & o \\
\hline Solanki et al., 2014 & - & ० & ० & ○ & o & & o \\
\hline Brofman et al. 2014 & ० & o & ० & - & o & & - \\
\hline Yang et al., 2014 & ○ & ० & ० & - & ० & 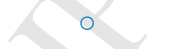 & o \\
\hline Pant et al., 2015 & ० & $\bullet$ & ० & ० & O & • & o \\
\hline Pizzuti et al., 2015 & $\circ$ & ○ & $\circ$ & 0 & 0 & $\bullet$ & $\bullet$ \\
\hline This paper & O & $\bullet$ & $\bullet$ & o & - & $\bullet$ & $\bullet$ \\
\hline
\end{tabular}

Table 1. Traceability frameworks on FSC from the literature 
GeoArea

Country, Region, Province, Area, Latitude, Longitude, SocioEconomicProfile, AgroProfile, LogisticProfile, InfrastructureProfile, ClimateProfile, EnvironmentalProfile, InfrastructureCostProfile, EnergyCostProfile, LaborCostProfile

GeoArea, Period, AgricultureValueAdded, CashSurplus, CurrentAccountalance, CentralGovernmentDebt,

SocioEconomicProfile ExportsOfGoodsAndServices, GDPperCapita, GDPGrowth, GrossCapitalFormation, ImportOfGoodAndServices, IndustryValueAdded, Inflation, PortfolioEquity, ServicesValueAdded, TradeInServices GeoArea, Period, \%AgriculturalIrrigatedLand, \%AgriculturalLand, AgriculturalValueAdded,

AgroProfile AgriculturalValueAddedForWorker, ArableLand, EmploymentInAgriculture, ForestArea, ImprovedWaterSource, LandArea, PermanentCropland, LandUnderCereal, RuralPopulation, CerealYield, LivestockProductionIndex

GeoArea, Period, FoodExport, FuelExport, TransportServices, TravelServices, AgriculturalRawMaterialImports, TariffRates, MerchandiseTrade, AbilityToTrackAndTraceConsignments, CompetenceAndQualityOfLogisticsServices,

LogisticProfile EaseOfArrangingCompetetivelyPricedShipment, TimeToExport, TimeToImport, EfficiencyOfCustomsClearanceProcess, FrequencyConsigneeWithExpectedTime

GeoArea, Period, AirTransportRegisteredCarriers, AnnualFreshWaterAgriculture, AnnualFreshWaterTotal,

InfrastructureProfile ContainerPortTraffic, ElectricPowerConsumption, ICTGoodsExport, ICTGoodsImport, InternetUsers, PassengerCars, RailLines, RenewableInternalFreshWater, RoadSectorFuelConsumption, RoadSectorEnergyConsumption, RoadsPaved, Vehicles

GeoAreaCode, Period, CO2Emissions, EnergyUse, MethaneEmissions, MortalityRateUnder5, NitrousOxideEmissions, OtherGreenhouseGasEmissions, PopulationGrowth, PopulationPrimaryCompletionRate, RatioOfGirlsToBoyInSchool,

ClimateProfile UranPopulation, AccessToElectricity, PovertyHeadcountRatio, PopulationInUrbbanAgglomerationOfMoreThanOneMillion, LandAreaWhereElevationIsBelow5m

GeoAreaCode, Period, AgriculturalMethaneEmissions, AgriculturalNitrousOxideEmissions, FishSpecies, MammalSpecies, CO2Emissions, ForestArea, MarineProtectedAreas, OrganicWaterPollutant, PlantSpecies, MethaneEmissions,

EnvironmentalProfile NitrousOxideEmissions, OtherGreenhouseGasEmissions, WaterPollutionFoodIndustry, WaterPollutionMetalIndustry, WaterPollutionPaperAndPulpIndustry, WaterPollutionTextileIndustry, WaterPollutionOtherIndustry

GeoAreaCode, AgricoltureCost, LicenseCost, ProductionBuildingCost, DistributionBuildingCost, EnergyInfrastructureCost,

InfrastructureCostProfile WoodlandCost

EnergyCostProfile GeoAreaCode, KWhElectricityCostDomestic, KWhElectricityCostIndustrial, KWhGasCostIndustrial, KwhSolarCost, KwhWindCost, KwhCarbonCost, KwhGasCost, FuelCost GeoAreaCode, OperatorCost, FarmerCost, WorkerCost, WarehouseWorkerCost, EmployeeCost, DriverCost

DemandGeoArea GeoArea, Period, Product, Demand, ChannelGDO, ChannelGrossMarket, ChannelGroceryShop, ChannelGroceryMarket

GeoAreaCode, ImportExportProfile, ProductCode, Period, TypeProduct, ImportFlow, InternalDemand, InternalProduction,

ImportExport ExportFlow

Supply

NodeCode, ProductCode, Period, NodeType, Country, ProductionQty, ProductionCost, PackagingCost, StorageCapacity, ProductionArea, LandfillCost, ProductionEmissions, TransformationEmissions, SupplierEmissions, LandfillEmissions

NodeCodeFrom, NodeCodeTo, FlowCount, GeoAreaFrom, GeoAreaTo, WeeklyConnections, Lines, Product,

Flow NominalCapacity, MaxCapacity, OverallFlow, LiquidBulk, DryBulk, AgroLiquidBulk, AgroDryBulk, GeneralCargo

EnterpriseCode, NodeCode, NodeType, Enterprise, ZipCode, Address, City, Province, Nation, Region, Continent,

Node RoadAccess, RailAccess, SeaAccess, AirAccess, GeoArea

EnterpriseCode, NodeCode, NodeType, NodeName, Enterprise, ZipCode, RoadAccess, ThroughputCapacity, Power,

Crop EnergyReq, WaterReq, CO2, Turnover, AgroProfile, SoilProfile, ClimateProfile, GeoArea

ProcessingPlant EnterpriseCode, NodeCode, NodeType, NodeName, Enterprise, ZipCode, RoadAccess, RailAccess, ThroughputCapacity, Power, EnergyReq, WaterReq, CO2, Turnover, WHProfile, LayoutProfile, GeoArea 
Market

Port

Terminal

Multimodal Hub

Rail Connection

Order

Product

DemandNode

Shelflife

Price

Shipment

TransportationMode

Package

UnitLoad

Enterprise

ShipmentProfile

Analysis

Simulation

EnterpriseCode, NodeCode, NodeType, NodeName, Enterprise, ZipCode, RoadAccess, TotalDemand, GrossMarket, Market, ConsumptionPoint, GeoArea

NodeCode, NodeName, NodeType, City, Province, Country, Region, Continent, TotalArea, RailLength, QuayLength,

Cranes, Employees, Vessels, Capacity, Throughput, MaxDraught, StorageArea, Totalterminals, Cruises, DryBulk,

LiquidBulk, GeneralCargo, Oilpipe, DryAgroBulk, LiquidAgroBulk, TranshipmentFraction, EUNorthernArea,

EUSouthernAREA, MediterraneanArea, ConnectionRail, ConnectionRoad, ConnectionMatirime

NodeCode, PortCode, TerminalCode, Type, Name, ProductCategory, Throughput, Period, Cranes, StraddleCarriers, Forklift, PortTruck, PipePlugs, ReeferfPlugs, VesselBerths, RailTerminal, Reachstakers, VesselCapacity, Area, StorageCapacity, throughputRate, LoadingRate, UnloadingRate, Temperatresotrage, CoolStorage, FrozenStorage

NodeCode, Nodename, NodeType, City, TotalArea, RailLemgth, Employees, StorageArea, StorageAreaTemp, TotalTerminals, DryBulk, LiquidBulk, GeneralCargo, OilPipe, DryArgoBulk, EUNorthernArea, EUSouthernArea, MediterraneanArea, ConnectionRail, ConnectionRoad, ConnectionMaritime, FixedCranes, MobileCranes, FloatingCranes ConnectionCode, Destination, Enterprise, Frequency, TravelHours, NodeCode, NodeName

OrderCode, Category, ShipmentCode, NodeCode, NodeCodeTo, DeliveryDate, PdkQuantity, PdkWeight, PalletQuantity, Parts, TransportationModeCode, Load, Description, ShipmentCode, UnitloadCode

ProductCode, ScientificaName, FoodCode, Category, Name, Variety, Brand, Enterprise, DOP, IGP, IGT, DOC, DOCG,

Energy, Edibility, Water, Protein, Lipid, Cholesterol, Carbohydrates, Amid, Sugar, Fibre, Alcol, Sodium, Potassium, Iron,

Calcium, Selenium, Tiamin, Riboflavin, VitaminA, VitaminB, VitaminC, VitaminE, EthyleneProduction,

525

EthyleneSensibility, ColdSensibility, HarvestPeriod, Certification, OptimalStorageTemperature, OptimaleStorageRh,

MaximumShelflife, PackageCode

ProductCode, NodeCode, Period, Quantity, Udm

ProductCode, TempMin, TempMax, Name, Type, RhMin, RhMax, ShelfLifeMin, ShelfLifeMax

ProductCode, Node, Period, Type, ProductName, Variety, Country, region, City, PriceGrossMarket, Udm, Week,

BeginDate, EndDate, Month, Market, ShopVariancy, DiscountVariancy, LargeRetailerVaiancy, PublicMarketVariancy,

RetailerVariancy

TransportationCode, ShipmentCode, NodeName, NodeCode, NodeCodeTo, ProducerCode, ShipperCode, CarrierCode, ImporterCode, CustomerCode, ContainerType, ContainerSize, ProductCode, CartonQty, UnitLoadQty, OriginPort, DestinationPort, DepartureDate, ArrivalDate

TransportationCode, TransportationName, TransportationType, VehicleName, Description, Class, LicenceRequired, VehicleLenght, VehicleWidth, LoadHeight, WeightCapacity, GrossTonnage, Co, Hc, Nox, Pm, Ch4, N2O, Nh3, SO2, CO2, CO2Eq, FuelConsumption, EnergyConsumption, TransportationCost, ContainerQuantity, VehicleQty, AvgSpeed, MaxDistance

PackageCode, ProductCode, PackageName, PackageType, PackageLevel, Firm, Shape, ProductWeight, PackageLength, PachageWidth, PAckageHeight, Udm, Cork, Sleever, Material, color, Weight, Barcode, PackagePrimary Province, Region, Nation, Continent

ShipmentCode, Ibutton, DatePeriod, Position, Temperature, ThermalKit, ContainerCode, Sampling, ShipmentCode, NodeName, NodeCode, NodeCodeTo, TransportationCode, EnterpriseCode

SimulationCode, ShipmentCode, ProductCode, TotalSulphurousDioxide, FreeSulphurousDioxide, OpticDensity420nm, TotalAcidity, VolatilAcidity, PhenolicAcid, SO2Free, SO2Total, ColorIntensity, SmellIntensity, TasteIntensity, Shipment, Ibutton, DatePeriod

SimulationCode, ShipmentCode, Product, SimStart, SimEnd, SmplingPeriod, Samplingtime, TempOffset, RhOffset, TempCurrent, RhCurrent, Shipment, Ibutton, DatePeriod

GlobalRadiation, Precipitation, RelativeHumidity, StationInfo, SunshineFraction, SunshineHours, TempAver,

ClimateProfileMonitoring TempDayTime, TempMeanMax, TempMeanMin, TempNihtTime, VapourPressure, WindSpeed

Demand, DewPoint, Distance, Ea_ed, EnergyCost, Eta, GeoArea, InfrastructureCost, LaborCost, Land, LandUse,

LogisticsCost, Node, OutputFlow, OutputLand, PET, Product, Rse, Soil, Transportation, Use, UseProductLimit,

$\underline{\text { SoilProfileMonitoring }}$

UseProductPercentage, UseProductRate, WaterRetention, ym

19978

Table 2. Database fields, abstraction levels (AL), and collected records. 
ACCEPTED MANUSCRIPT

\begin{tabular}{|c|c|c|c|c|c|}
\hline Type of metric & KPIs & Unit of measure & \multicolumn{2}{|c|}{ Distribution Scenarios } & \multirow{2}{*}{$\begin{array}{l}\text { Change } \\
\Delta \%\end{array}$} \\
\hline \multirow{4}{*}{ Logistics } & & & As-is & To-be & \\
\hline & Number of Shipments & (shipments/year) & 2,867 & 2,665 & $-7 \%$ \\
\hline & Total travelling & (km/year) & $2,167,702.76$ & $1,938,336.77$ & $-11 \%$ \\
\hline & Average Travel Time & (hour/shipment) & 22.17 & 21.88 & $-1 \%$ \\
\hline \multirow[b]{2}{*}{ Economic } & Average Transport Cost & (€/shipment) & 466.16 & 449.47 & $-4 \%$ \\
\hline & Fuel consumption & (kg/year) & $29,516,592.60$ & $26,894,936.30$ & $-9 \%$ \\
\hline \multirow{9}{*}{ Environmental } & Equivalent $\mathrm{CO}_{2}\left(\mathrm{GWP}_{100}\right)$ & (kg/year) & $900,470.15$ & $823,989.13$ & $-8 \%$ \\
\hline & $\mathrm{HC}$ & (kg/year) & 237.005 & 165.073 & $-30 \%$ \\
\hline & $\mathrm{NOx}$ & (kg/year) & $11,828.91$ & $11,492.32$ & $-3 \%$ \\
\hline & PM & (kg/year) & 384.591 & 365.924 & $-5 \%$ \\
\hline & $\mathrm{CH}_{4}$ & (kg/year) & 39.990 & 37.847 & $-5 \%$ \\
\hline & $\mathrm{N}_{2} \mathrm{O}$ & (kg/year) & 37.833 & 30.257 & $-20 \%$ \\
\hline & $\mathrm{NH}_{3}$ & (kg/year) & 3.87 & 2.75 & $-29 \%$ \\
\hline & $\mathrm{SO}_{2}$ & (kg/year) & 3432.387 & 3431.876 & n.s. \\
\hline & $\mathrm{CO}_{2}$ & (kg/year) & $892,037.37$ & $817,939.70$ & $-8 \%$ \\
\hline
\end{tabular}

Table 3. What-if analysis with the Company testbed. 


\section{ACCEPTED MANUSCRIPT}

\begin{tabular}{|c|c|c|c|c|c|c|c|}
\hline Reference & $\begin{array}{c}\text { IT } \\
\text { Architecture }\end{array}$ & $\begin{array}{c}\text { Data } \\
\text { Integration \& } \\
\text { Alignment }\end{array}$ & $\begin{array}{l}\text { Regulation \& } \\
\text { standard } \\
\text { compliance }\end{array}$ & $\begin{array}{c}\text { Tracking } \\
\text { Technologies }\end{array}$ & $\begin{array}{l}\text { Food } \\
\text { Safety }\end{array}$ & $\begin{array}{l}\text { Supply Chain } \\
\text { Design \& } \\
\text { Management }\end{array}$ & $\begin{array}{c}\text { Support to } \\
\text { decision- } \\
\text { making }\end{array}$ \\
\hline Bechini et al., 2005 & $\bullet$ & $\bullet$ & ० & ○ & 0 & O & o \\
\hline Shanahan et al., 2009 & ० & ○ & - & - & ० & ० & o \\
\hline Lenton, 2010 & ० & ○ & - & & $\bullet$ & ० & o \\
\hline Costa et al., 2013 & - & ० & ० & - & ० & ० & ० \\
\hline Storøy et al, 2013 & - & $\bullet$ & ० & ○ & O & ○ & o \\
\hline Zhang et al., 2014 & ○ & ० & - & ○ & $\bullet$ & ○ & o \\
\hline Solanki et al., 2014 & - & ० & ० & ○ & o & & o \\
\hline Brofman et al. 2014 & ० & o & ० & - & o & & - \\
\hline Yang et al., 2014 & ○ & ० & ० & - & ० & 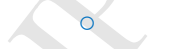 & o \\
\hline Pant et al., 2015 & ० & $\bullet$ & ० & ० & O & • & o \\
\hline Pizzuti et al., 2015 & $\circ$ & ○ & $\circ$ & 0 & 0 & $\bullet$ & $\bullet$ \\
\hline This paper & O & $\bullet$ & $\bullet$ & o & - & $\bullet$ & $\bullet$ \\
\hline
\end{tabular}

Table 1. Traceability frameworks on FSC from the literature 


\section{$\frac{\text { Table }}{\text { GeoArea }}$}

GeoArea, Period, AgricultureValueAdded, CashSurplus, CurrentAccountalance, CentralGovernmentDebt, ExportsOfGoodsAndServices, GDPperCapita, GDPGrowth, GrossCapitalFormation, ImportOfGoodAndServices, IndustryValueAdded, Inflation, PortfolioEquity, ServicesValueAdded, TradeInServices

GeoArea, Period, \%AgriculturalIrrigatedLand, \%AgriculturalLand, AgriculturalValueAdded, AgriculturalValueAddedForWorker, ArableLand, EmploymentInAgriculture, ForestArea, ImprovedWaterSource, LandArea, PermanentCropland, LandUnderCereal, RuralPopulation, CerealYield, LivestockProductionIndex

GeoArea, Period, FoodExport, FuelExport, TransportServices, TravelServices, AgriculturalRawMaterialImports, TariffRates,

MerchandiseTrade, AbilityToTrackAndTraceConsignments, CompetenceAndQualityOfLogisticsServices,

LogisticProfile EaseOfArrangingCompetetivelyPricedShipment, TimeToExport, TimeToImport, EfficiencyOfCustomsClearanceProcess, FrequencyConsigneeWithExpectedTime

GeoArea, Period, AirTransportRegisteredCarriers, AnnualFreshWaterAgriculture, AnnualFreshWaterTotal, ContainerPortTraffic,

InfrastructureProfile ElectricPowerConsumption, ICTGoodsExport, ICTGoodsImport, InternetUsers, PassengerCars, RailLines, RenewableInternalFreshWater, RoadSectorFuelConsumption, RoadSectorEnergyConsumption, RoadsPaved, Vehicles

GeoAreaCode, Period, CO2Emissions, EnergyUse, MethaneEmissions, MortalityRateUnder5, NitrousOxideEmissions,

OtherGreenhouseGasEmissions, PopulationGrowth, PopulationPrimaryCompletionRate, RatioOfGirlsToBoyInSchool, UranPopulation, AccessToElectricity, PovertyHeadcountRatio, PopulationInUrbbanAgglomerationOfMoreThanOneMillion, LandAreaWhereElevationIsBelow5m

GeoAreaCode, Period, AgriculturalMethaneEmissions, AgriculturalNitrousOxideEmissions, FishSpecies, MammalSpecies, CO2Emissions, ForestArea, MarineProtectedAreas, OrganicWaterPollutant, PlantSpecies, MethaneEmissions, NitrousOxideEmissions, OtherGreenhouseGasEmissions, WaterPollutionFoodIndustry, WaterPollutionMetalIndustry, WaterPollutionPaperAndPulpIndustry, WaterPollutionTextileIndustry, WaterPollutionOtherIndustry

InfrastructureCostProfile GeoAreaCode, AgricoltureCost, LicenseCost, ProductionBuildingCost, DistributionBuildingCost, EnergyInfrastructureCost, WoodlandCost

EnergyCostProfile GeoAreaCode, KWhElectricityCostDomestic, KWhElectricityCostIndustrial, KWhGasCostIndustrial, KwhSolarCost, KwhWindCost, KwhCarbonCost, KwhGasCost, FuelCost

LaborCostProfile

DemandGeoArea

Expense GeoAreaCode, OperatorCost, FarmerCost, WorkerCost, WarehouseWorkerCost, EmployeeCost, DriverCost

Expense

GeoArea, Period, Product, Demand, ChannelGDO, ChannelGrossMarket, ChannelGroceryShop, ChannelGroceryMarket

GeoArea, Period, Product, ExpensePerFamily

GeoAreaImporter, GeoAreaExporter, National tax, Import rules

ExportRules

GeoAreaImporter, GeoAreaExporter, Export rules

ImportExport GeoAreaCode, ImportExportProfile, ProductCode, Period, TypeProduct, ImportFlow, InternalDemand, InternalProduction, ExportFlow

Supply NodeCode, ProductCode, Period, NodeType, Country, ProductionQty, ProductionCost, PackagingCost, StorageCapacity, ProductionArea, LandfillCost, ProductionEmissions, TransformationEmissions, SupplierEmissions, LandfillEmissions

NodeCodeFrom, NodeCodeTo, FlowCount, GeoAreaFrom, GeoAreaTo, WeeklyConnections, Lines, Product, NominalCapacity, MaxCapacity, OverallFlow, LiquidBulk, DryBulk, AgroLiquidBulk, AgroDryBulk, GeneralCargo

Flow EnterpriseCode, NodeCode, NodeType, Enterprise, ZipCode, Address, City, Province, Nation, Region, Continent, RoadAccess, RailAccess, SeaAccess, AirAccess, GeoArea

Node

EnterpriseCode, NodeCode, NodeType, NodeName, Enterprise, ZipCode, RoadAccess, ThroughputCapacity, Power, EnergyReq,

Crop WaterReq, CO2, Turnover, AgroProfile, SoilProfile, ClimateProfile, GeoArea

EnterpriseCode, NodeCode, NodeType, NodeName, Enterprise, ZipCode, RoadAccess, RailAccess, ThroughputCapacity, Power, EnergyReq, WaterReq, CO2, Turnover, WHProfile, LayoutProfile, GeoArea EnterpriseCode, NodeName, Enterprise, Zipcode, RoadAccess, RailAccess, StorageCapacity, HandlingCapacity, Power, EnergyReq,

StorageFacility WaterReq, CO2, Turnover, WHProfile, LayoutProfile, PlantProfile, BmHeight, BmDepth, BmLayer, UlBmLayer, RackLevel, BeamCode, ColumnCode, CrossCode, FwLevel, UlCode, GeoArea

Market $\quad$ EnterpriseCode, NodeCode, NodeType, NodeName, Enterprise, ZipCode, RoadAccess, TotalDemand, GrossMarket, Market, ConsumptionPoint, GeoArea

NodeCode, NodeName, NodeType, City, Province, Country, Region, Continent, TotalArea, RailLength, QuayLength, Cranes, Employees, Vessels, Capacity, Throughput, MaxDraught, StorageArea, Totalterminals, Cruises, DryBulk, LiquidBulk, GeneralCargo, Oilpipe,

Port DryAgroBulk, LiquidAgroBulk, TranshipmentFraction, EUNorthernArea, EUSouthernAREA, MediterraneanArea, ConnectionRail, ConnectionRoad, ConnectionMatirime

NodeCode, PortCode, TerminalCode, Type, Name, ProductCategory, Throughput, Period, Cranes, StraddleCarriers, Forklift, PortTruck,

Terminal PipePlugs, ReeferfPlugs, VesselBerths, RailTerminal, Reachstakers, VesselCapacity, Area, StorageCapacity, throughputRate, LoadingRate, UnloadingRate, Temperatresotrage, CoolStorage, FrozenStorage

NodeCode, Nodename, NodeType, City, TotalArea, RailLemoth, Employees, StorageArea, StorageAreaTemp, TotalTerminals, DryBulk, LiquidBulk, GeneralCargo, OilPipe, DryArgoBulk, EUNorthernArea, EUSouthernArea, MediterraneanArea, ConnectionRail,

$\begin{array}{ll}\text { Multimodal Hub } & \text { LiquidBulk, GeneralCargo, OilPipe, Dry ArgoBulk, EUNorthernArea, EUSouthernArea, M } \\ & \text { ConnectionRoad, ConnectionMaritime, FixedCranes, MobileCranes, FloatingCranes } \\ \text { Rail Connection } & \text { ConnectionCode, Destination, Enterprise, Frequency, TravelHours, NodeCode, NodeName }\end{array}$

ConnectionCode, Destination, Enterprise, Frequency, TravelHours, NodeCode, NodeName

\begin{tabular}{ll}
\hline Order & OrderCode, Category, ShipmentCode, NodeCode, NodeCodeTo, DeliveryDate, PdkQuantity, PdkWeight, PalletQuantity, Parts, \\
& TransportationModeCode, Load, Description, ShipmentCode, UnitloadCode \\
& ProductCode, ScientificaName, FoodCode, Category, Name, Variety, Brand, Enterprise, DOP, IGP, IGT, DOC, DOCG, Energy, Edibility,
\end{tabular}

\begin{tabular}{ll}
\hline Order & OrderCode, Category, ShipmentCode, NodeCode, NodeCodeTo, DeliveryDate, PdkQuantity, PdkWeight, PalletQuantity, Parts, \\
& TransportationModeCode, Load, Description, ShipmentCode, UnitloadCode \\
& ProductCode, ScientificaName, FoodCode, Category, Name, Variety, Brand, Enterprise, DOP, IGP, IGT, DOC, DOCG, Energy, Edibility,
\end{tabular}

Water, Protein, Lipid, Cholesterol, Carbohydrates, Amid, Sugar, Fibre, Alcol, Sodium, Potassium, Iron, Calcium, Selenium, Tiam
Riboflavin, VitaminA, VitaminB, VitaminC, VitaminE, EthyleneProduction, EthyleneSensibility, ColdSensibility, HarvestPeriod, Certification, OptimalStorageTemperature, OptimaleStorageRh, MaximumShelflife, PackageCode ProductCode, NodeCode, Period, Quantity, Udm

DemandNode

Shelflife

ProductCode, NodeCode, Period, Quantity, Udm

Shelflife

ProductCode, TempMin, TempMax, Name, Type, RhMin, RhMax, ShelfLifeMin, ShelfLifeMax

ProductCode, Node, Period, Type, ProductName, Variety, Country, region, City, PriceGrossMarket, Udm, Week, BeginDate, EndDate, Month, Market, ShopVariancy, DiscountVariancy, LargeRetailerVaiancy, PublicMarketVariancy, RetailerVariancy

TransportationCode, ShipmentCode, NodeName, NodeCode, NodeCodeTo, ProducerCode, ShipperCode, CarrierCode, ImporterCode,

Shipment CustomerCode, ContainerType, ContainerSize, ProductCode, CartonQty, UnitLoadQty, OriginPort, DestinationPort, DepartureDate, ArrivalDate

TransportationCode, TransportationName, TransportationType, VehicleName, Description, Class, LicenceRequired, VehicleLenght,

TransportationMode VehicleWidth, LoadHeight, WeightCapacity, GrossTonnage, Co, Hc, Nox, Pm, Ch4, N2O, Nh3, SO2, CO2, CO2Eq, FuelConsumption, EnergyConsumption, TransportationCost, ContainerQuantity, VehicleQty, AvgSpeed, MaxDistance

Package

PackageCode, ProductCode, PackageName, PackageType, PackageLevel, Firm, Shape, ProductWeight, PackageLength, PachageWidth, PAckageHeight, Udm, Cork, Sleever, Material, color, Weight, Barcode, PackagePrimary

UnitLoad UnitLoadCode, UnitLoadLenght, UnitLoadWidth, UnitLoadHeight, unitLoadWeight, Material

Enterprise $\quad$ EnterpriseCode, NodeName, Group, Enterprise, NodeType, Importer, Exporter, Grower, Distributor, Address, Zipcode, City, Province, Region, Nation, Continent

ShipmentProfile

ShipmentCode, Ibutton, DatePeriod, Position, Temperature, ThermalKit, ContainerCode, Sampling, ShipmentCode, NodeName, NodeCode, NodeCodeTo, TransportationCode, EnterpriseCode

Analysis

SimulationCode, ShipmentCode, ProductCode, TotalSulphurousDioxide, FreeSulphurousDioxide, OpticDensity420nm, TotalAcidity, VolatilAcidity, PhenolicAcid, SO2Free, SO2Total, ColorIntensity, SmellIntensity, TasteIntensity, Shipment, Ibutton, DatePeriod

Simulation SimulationCode, ShipmentCode, Product, SimStart, SimEnd, SmplingPeriod, Samplingtime, TempOffset, RhOffset, TempCurrent, RhCurrent, Shipment, Ibutton, DatePeriod

ClimateProfileMonitoring Globaliation, Precipitation, RelativeHumidity, StationInfo, SunshineFraction, SunshineHours, TempAver, TempDayTime,

TempMeanMax, TempMeanMin, TempNihtTime, VapourPressure, WindSpeed

Demand, DewPoint, Distance, Ea_ed, EnergyCost, Eta, GeoArea, InfrastructureCost, LaborCost, Land, LandUse, LogisticsCost, Node.

Table 2. Database fields, abstraction levels (AL), and collected records. 
ACCEPTED MANUSCRIPT

\begin{tabular}{|c|c|c|c|c|c|}
\hline Type of metric & KPIs & Unit of measure & \multicolumn{2}{|c|}{ Distribution Scenarios } & \multirow{2}{*}{$\begin{array}{l}\text { Change } \\
\Delta \%\end{array}$} \\
\hline \multirow{4}{*}{ Logistics } & & & As-is & To-be & \\
\hline & Number of Shipments & (shipments/year) & 2,867 & 2,665 & $-7 \%$ \\
\hline & Total travelling & (km/year) & $2,167,702.76$ & $1,938,336.77$ & $-11 \%$ \\
\hline & Average Travel Time & (hour/shipment) & 22.17 & 21.88 & $-1 \%$ \\
\hline \multirow[b]{2}{*}{ Economic } & Average Transport Cost & (€/shipment) & 466.16 & 449.47 & $-4 \%$ \\
\hline & Fuel consumption & (kg/year) & $29,516,592.60$ & $26,894,936.30$ & $-9 \%$ \\
\hline \multirow{9}{*}{ Environmental } & Equivalent $\mathrm{CO}_{2}\left(\mathrm{GWP}_{100}\right)$ & (kg/year) & $900,470.15$ & $823,989.13$ & $-8 \%$ \\
\hline & $\mathrm{HC}$ & (kg/year) & 237.005 & 165.073 & $-30 \%$ \\
\hline & $\mathrm{NOx}$ & (kg/year) & $11,828.91$ & $11,492.32$ & $-3 \%$ \\
\hline & PM & (kg/year) & 384.591 & 365.924 & $-5 \%$ \\
\hline & $\mathrm{CH}_{4}$ & (kg/year) & 39.990 & 37.847 & $-5 \%$ \\
\hline & $\mathrm{N}_{2} \mathrm{O}$ & (kg/year) & 37.833 & 30.257 & $-20 \%$ \\
\hline & $\mathrm{NH}_{3}$ & (kg/year) & 3.87 & 2.75 & $-29 \%$ \\
\hline & $\mathrm{SO}_{2}$ & (kg/year) & 3432.387 & 3431.876 & n.s. \\
\hline & $\mathrm{CO}_{2}$ & (kg/year) & $892,037.37$ & $817,939.70$ & $-8 \%$ \\
\hline
\end{tabular}

Table 3. What-if analysis with the Company testbed. 
Decision-makers

Bureau of Transport an Infrastructures

Regulatory Agencies, Custon

Bureau of Agriculture and Environment
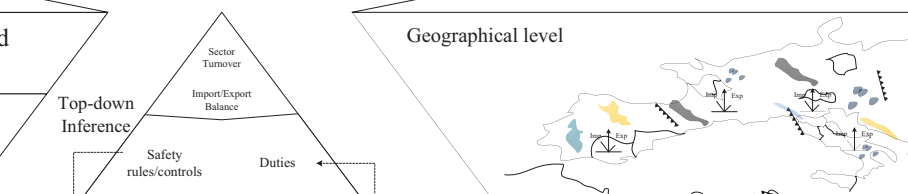

Port Authorities

Consumers

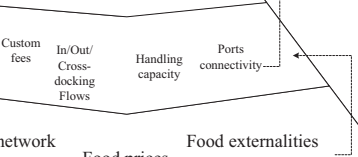

Retailers, Markets

Carriers, Distributors Producers, Suppliers Growers,
Farmers
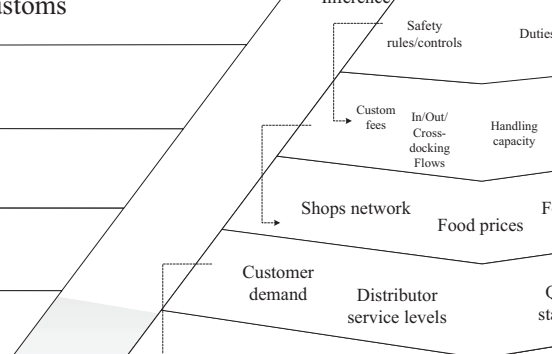

\section{Distributor} $\begin{array}{r}\begin{array}{r}\text { Distributor } \\ \text { service levels }\end{array} \\ \hline\end{array}$ food prices Food externalities Logistics level

Facility

S

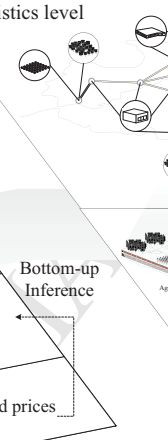
crice yed sevels yields Weather forecas Fertilizer cost Supplier demand Suppliers nodes

Data Architecture
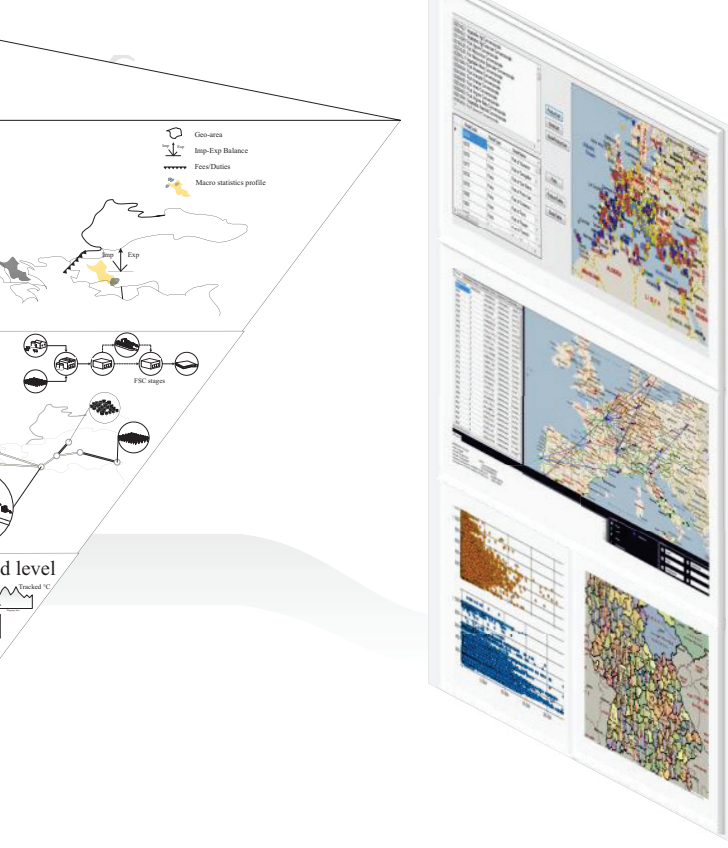

Decision-support Platform
FSC Planning targets

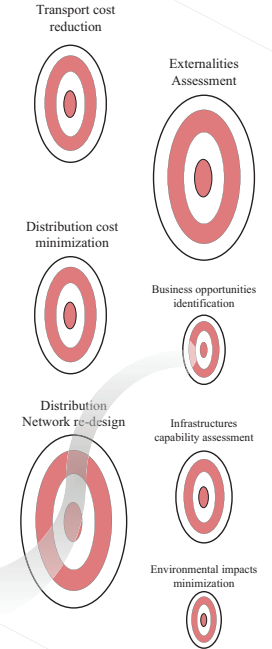

Fig. 1. Framework goals, boundaries and decision-flow 


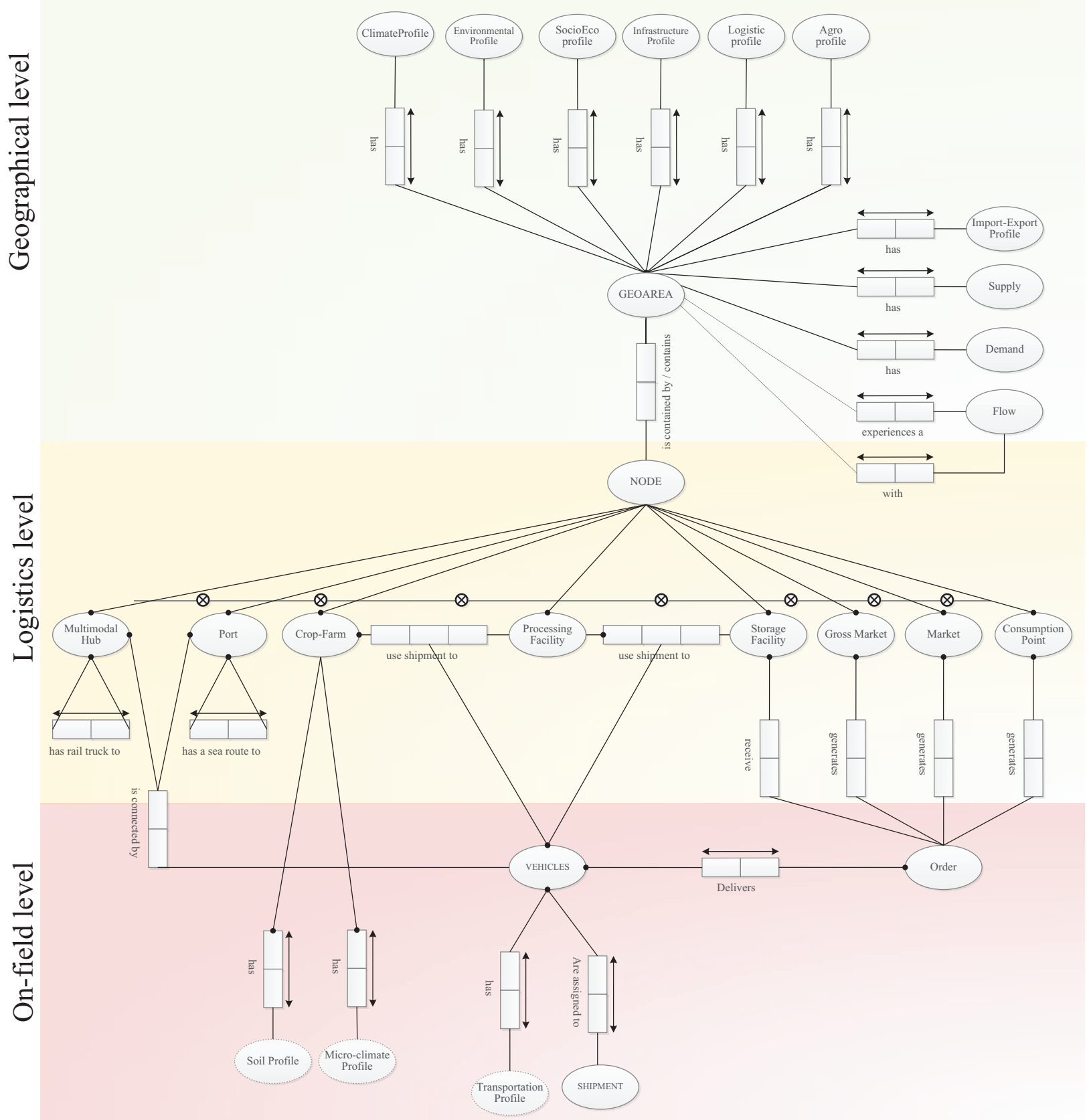

Fig. 2. Framework entities 


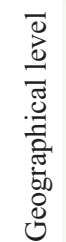

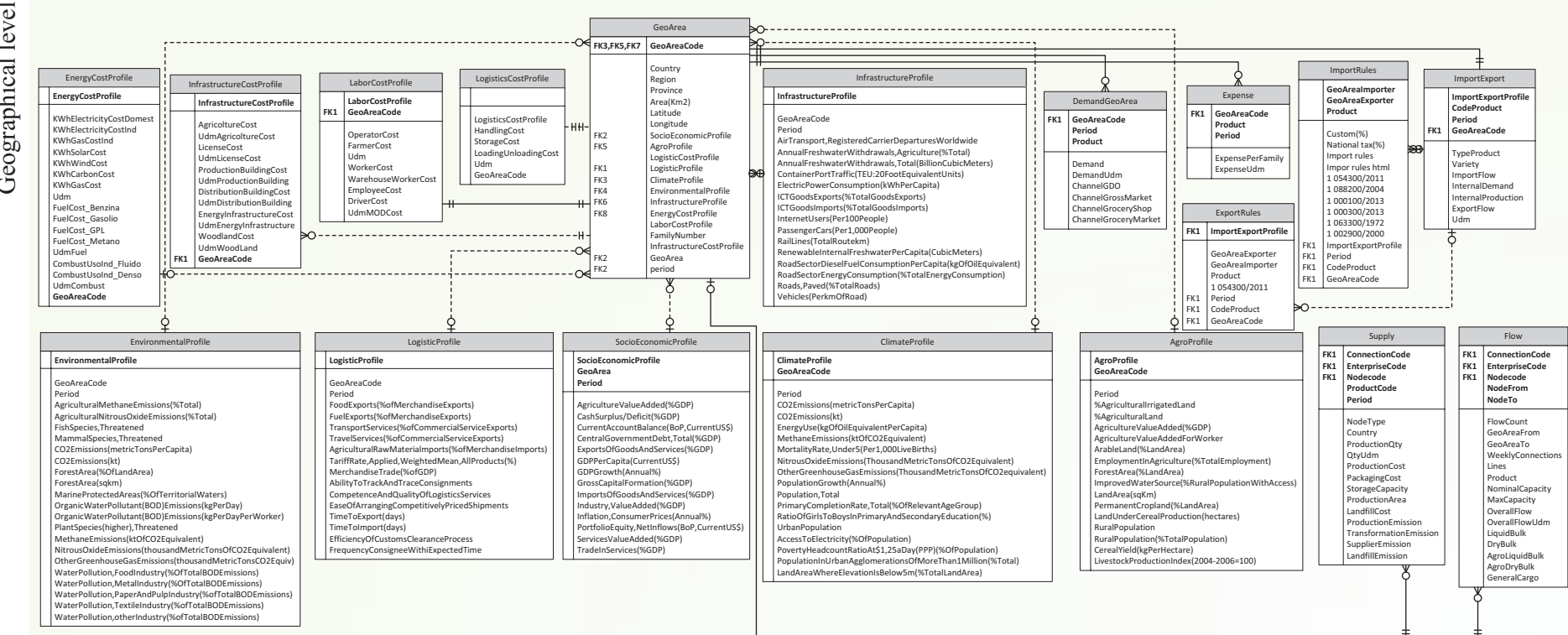

$\overline{0}$
0
0
0
0
0
0
0
0

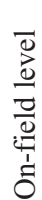
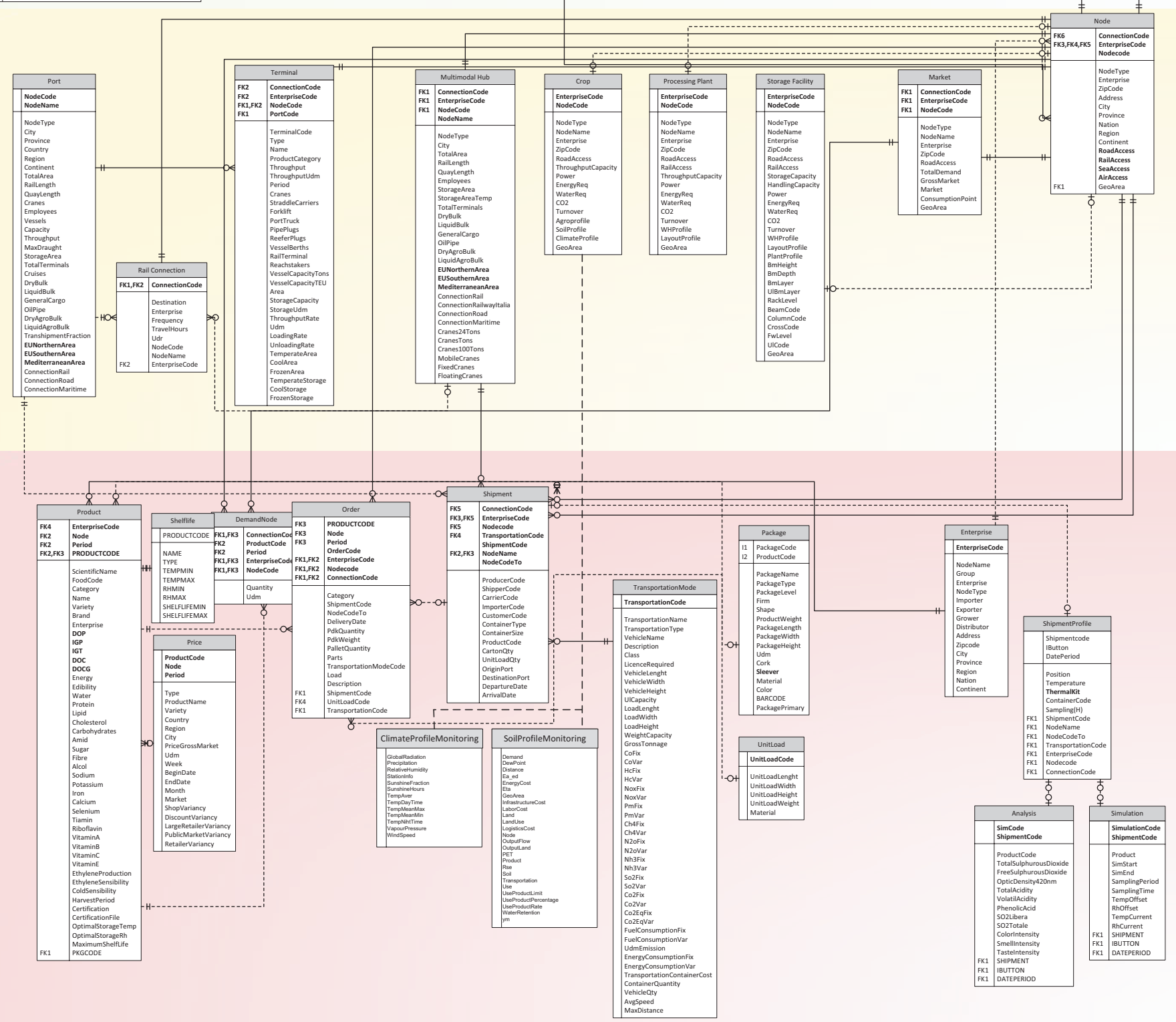

Fig. 3. Database E-R diagram. 


\section{ACCEPTED MANUSCRIPT}

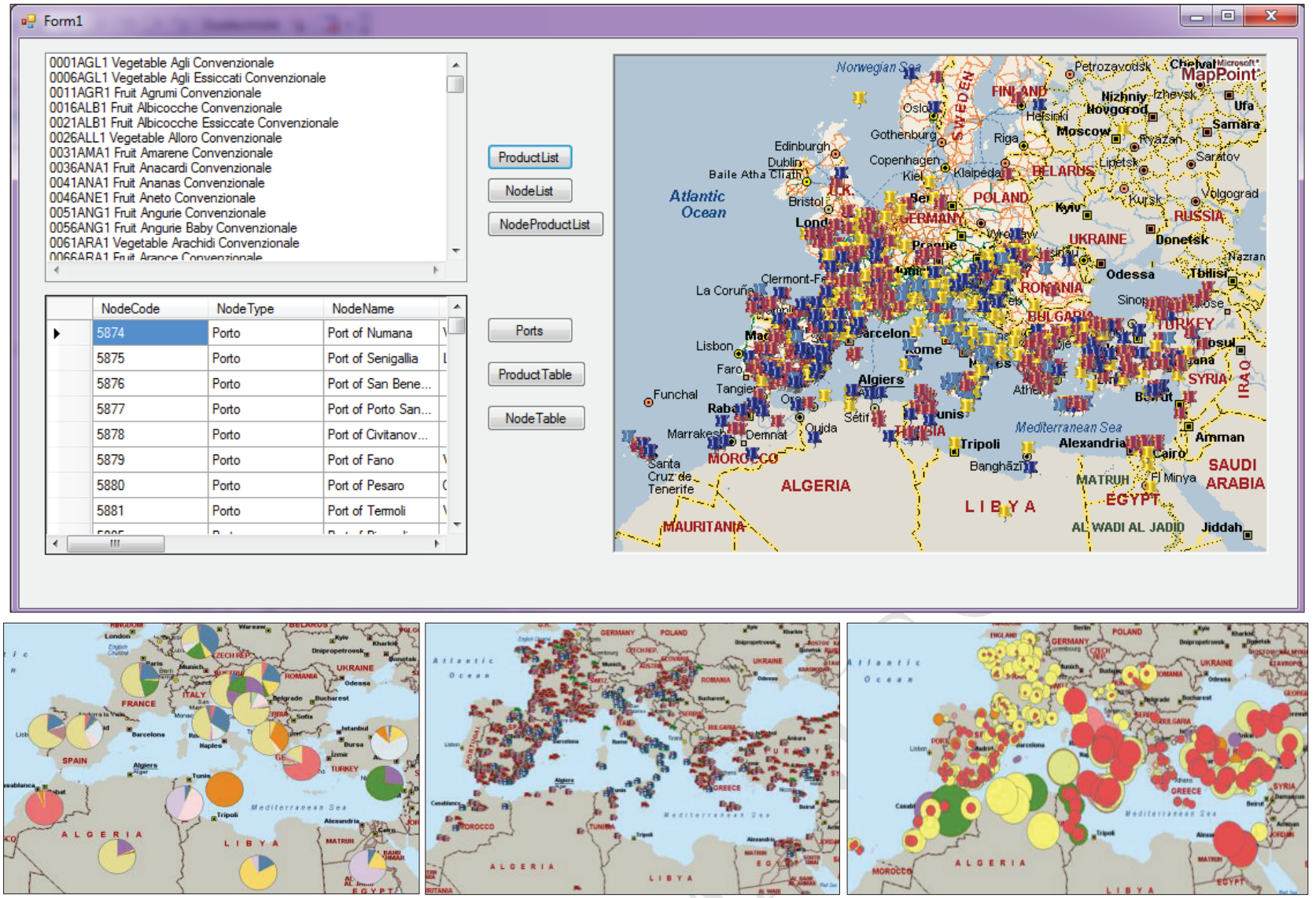

Fig. 4. Tool GUIs: Overviewing the high-level FSC records with a GIS. 

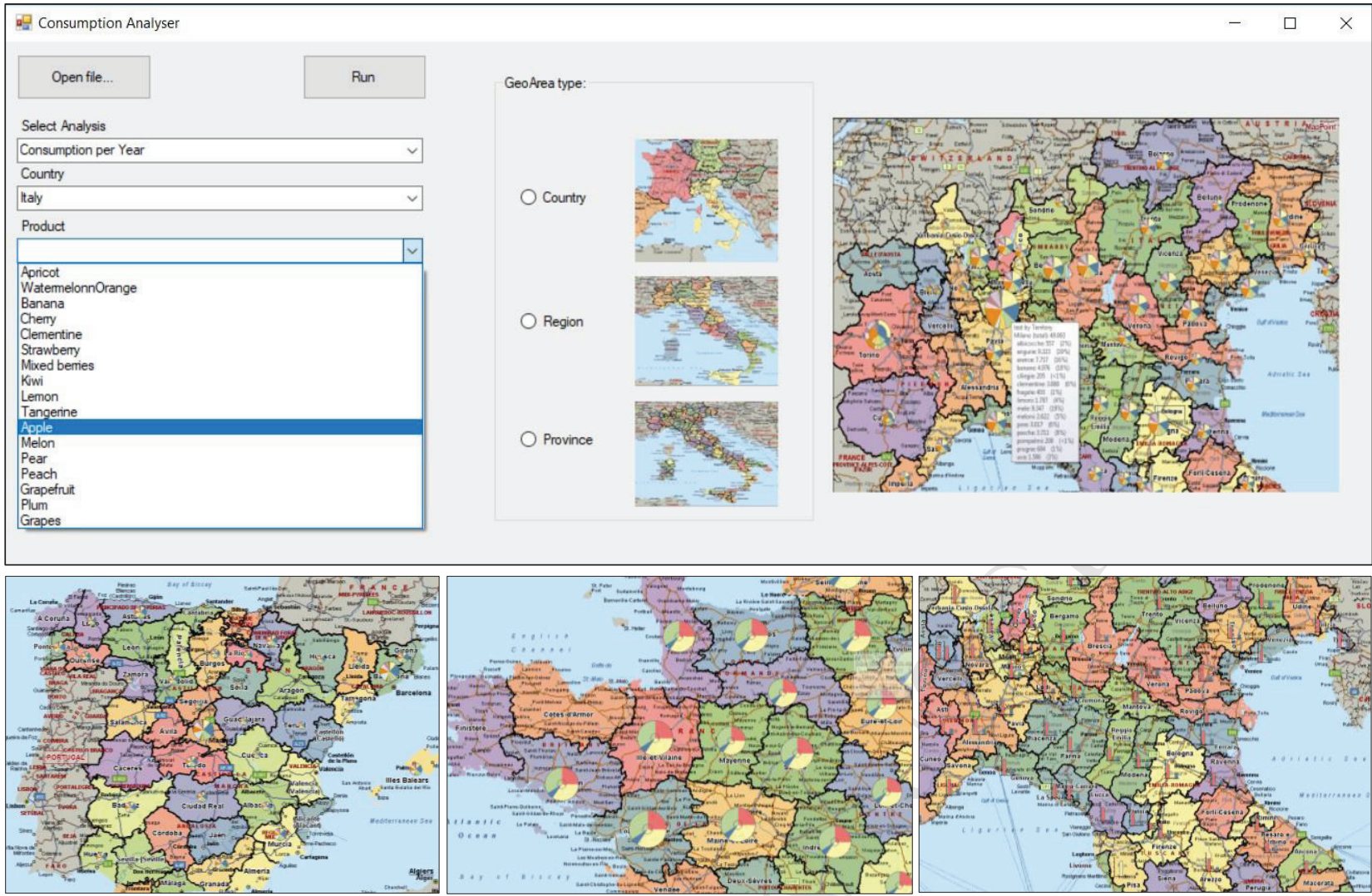

Fig. 5. Tool GUIs: Inferring data across different abstraction levels 

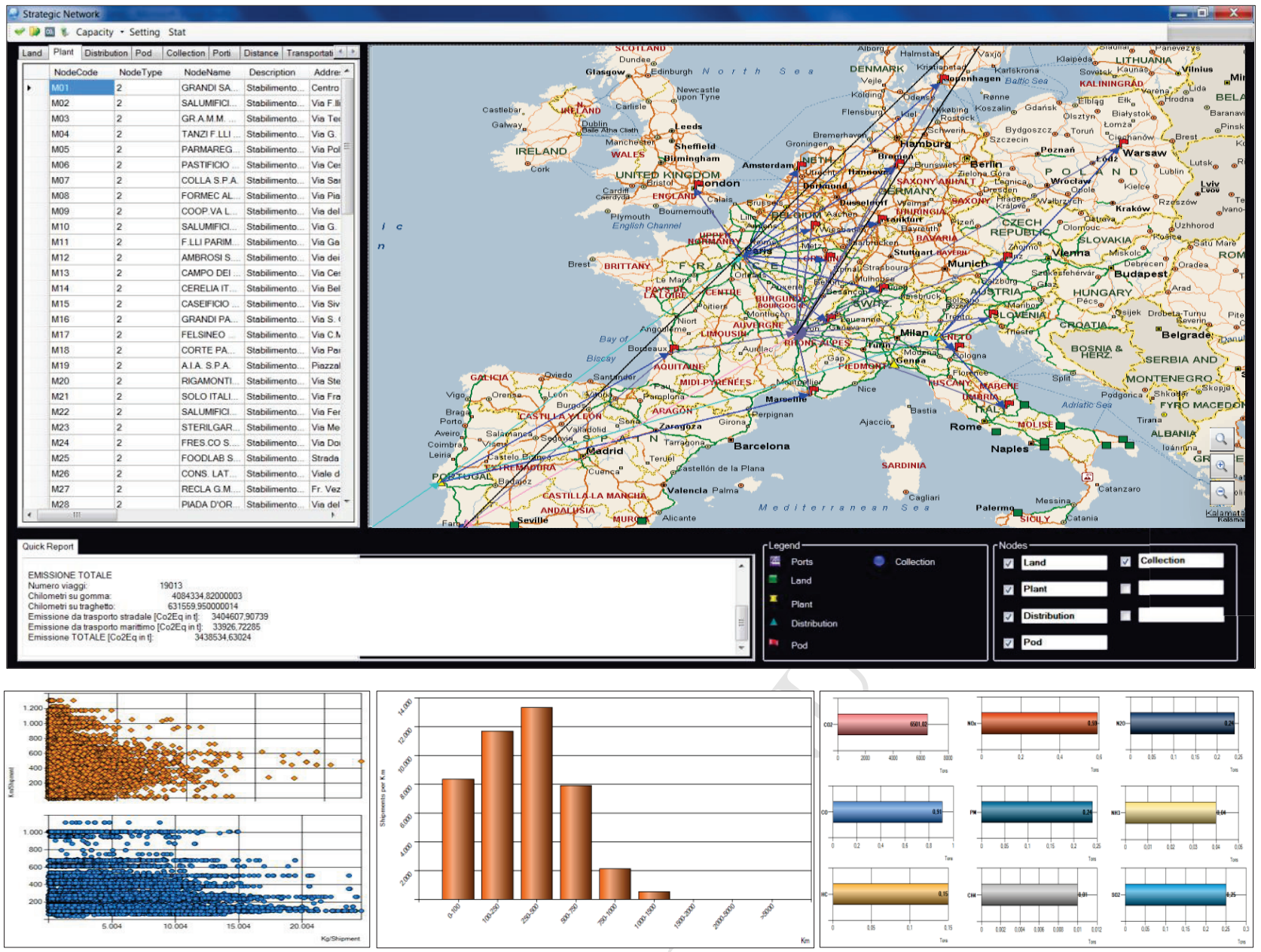

Fig. 6. Tool GUIs: Distribution scenarios and KPIs assessment. 


\section{ACCEPTED MANUSCRIPT}

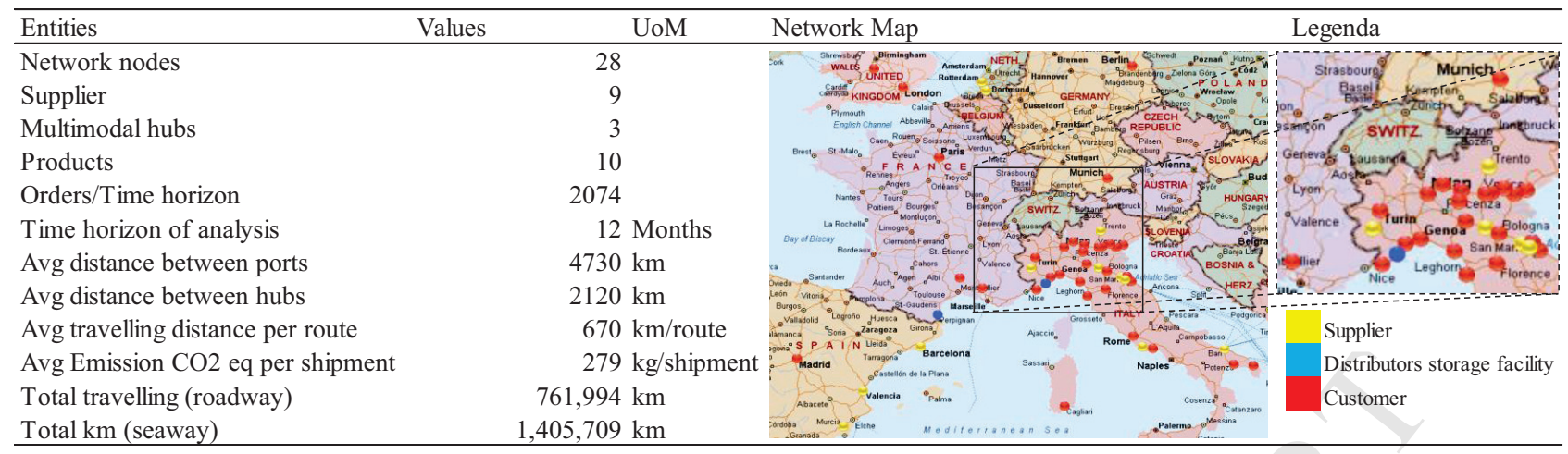

Fig. 7. Company's network features and map. 

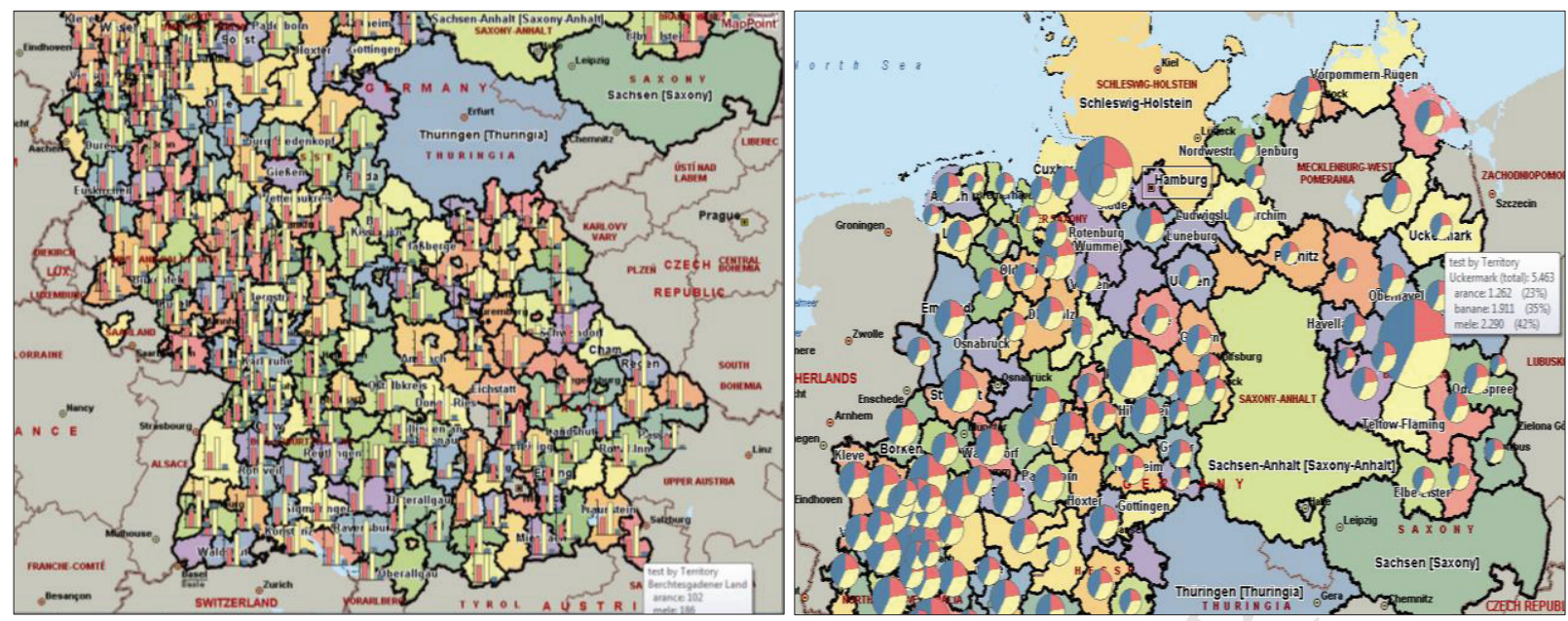

Fig. 8. Customer demand for oranges, peaches and apples in German markets. 


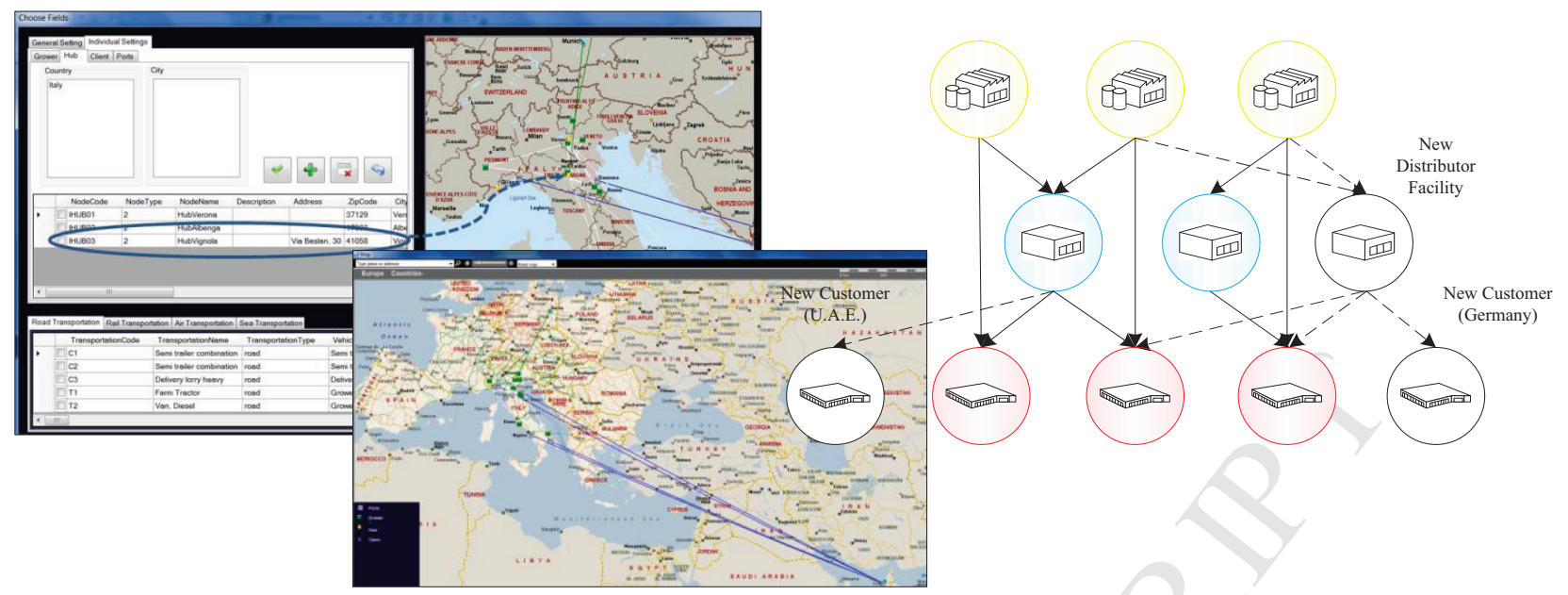

Fig. 9. Tool GUIs: Company testbed 


\section{$1 \quad$ Highlights}

2 This paper:

3 - is developed within the European Union and Mediterranean Project FUTUREMED;

4 - presents a framework for the implementation of data-driven analyses in sustainable food $5 \quad$ operations planning and management;

6 - addresses micro and macro aspects of the FSC and provides a hierarchical data architecture,

7 incorporated within a tailored decision-support platform;

8 - supports the design and planning of sustainable food production and distribution activities;

9

10 The illustrated database and tool:

11 - are validated with a case study of an Italian fruit trader considering opening a warehouse to serve new markets. 